QUALITATIVE THEORY OF DYNAMICAL SYSTEMS 1, 1-70 (1999)

ARTICLE NO. 1

\title{
A Survey of Isochronous Centers
}

\section{Javier Chavarriga}

\author{
Departament de Matemàtica. Escola Universitària Politècnica. Universitat de \\ Lleida. Avda. Jaume II, 69. Campus Cappont 25001. Lleida. SPAIN. \\ E-mail: chava@eup.udl.es \\ and \\ Marco Sabatini \\ Dipartimento di Matematica. Università degli Studi di Trento. \\ I-38050, Povo(TN), ITALY. \\ E-mail: sabatini@science.unitn.it
}

Key Words: Integrability, center-focus problem, isochronicity

\section{SUMMARY}

1. Introduction.

2. The center problem and the isochronicity problem.

3. Linearizations.

4. Computation of isochronous constants.

5. Commuting systems.

6. Complex systems.

7. Hamiltonian systems.

8. Uniformly isochronous centers.

9. Non-hamiltonian second order O.D.E's.

10. Quadratic systems.

11. Cubic systems with homogeneous nonlinearities.

12. Cubic reversible systems.

13. Polynomial systems with homogeneous nonlinearities.

14. Isochronous centers of a cubic system with degenerate infinity.

15. Kukles' system.

16. References. 


\section{INTRODUCTION}

In this survey we give an overview of the results obtained in the study of isochronous centers of vector fields in the plane. This paper consists of two parts. In the first one (sections $2-8$ ), we review some general techniques that proved to be useful in the study of isochronicity. In the second one (sections 9-16), we try to give a picture of the state of the art at the moment this review was written.

In section 2, we give some basic definitions about centers, isochronous centers, first integrals, integrating factors, particular algebraic solutions, and other related concepts. In this sections we also give some general theorems about centers and isochronous centers, and we give a brief account of the evolution of the researches in this field.

In the successive sections we focus on various methods that have been used in attacking the isochonicity problem. We start with linearizations in section 3, stating Poincaré's classical theorem and some of its consequences. Section 4 is devoted to describe the procedure that leads to define and compute isochronous constants. In section 5, commutators are introduced, and basic facts about couples of commuting systems are described. Classical theorems about systems obtained from complex ordinary differential equations are collected in section 6 . Hamiltonian systems are considered in section 7, where their connection to the study of the Jacobian Conjecture is showed, too. Section 8 is concerned with systems having constant angular speed with respect to some coordinate system.

The second part starts with section 9, that is devoted to recent results about second order differential equations not immediately reducible to hamiltonian systems. This section also contains the characterization of isochronous centers of reversible Liénard systems. In section 10 we list all fundamental results about isochronous centers of quadratic systems. Next section contains results about cubic systems with homogeneous nonlinearities. Sections 12 is devoted to cubic reversible systems. In section 13 we collect results about quartic and quintic systems with homogeneous nonlinearities. A class of particular cubic systems, with degenerate infinity is considered in section 14. Finally, section 15 is devoted to Kukles system.

All the sections of the second part, and some of the first part, contain tables, where the main features of the considered systems are collected. When possible, for every class of systems we have written the system in rectangular and polar coordinates, and we have reported a first integral, a commutator, a linearization and a reciprocal integrating factor.

The bibliography contains references both to papers devoted to the study of isochronicity and to papers concerned with integrability of plane systems and the study of the period function of centers. We have tried to make the bibliography so complete as possible for what is concerned with 
isochronicity. We have made no effort to make it complete for papers about integrability and the study of the period function. We address the reader interested in integrability problems to the forthcoming review paper by Conti [37].

We apologize for possible mistakes and encourage the readers to communicate us any corrections. ${ }^{1}$

\section{THE CENTER PROBLEM AND THE ISOCHRONICITY PROBLEM}

We consider here planar vector fields or equivalently systems of differential equations

$$
\frac{d x}{d t}=\dot{x}=X(x, y), \quad \frac{d y}{d t}=\dot{y}=Y(x, y),
$$

with $(x, y) \in U$, open connected subset of $\mathbf{R}^{2}$, and $X, Y \in C^{k}(U, \mathbf{R})$, with $k \geq 1$. Poincaré in [78] defined the notion of center for a real system on the plane.

DEFINITION 2.1. We say that an isolated singular point $O$ of (1) is a center if and only if there exists a punctured neighbourhood $V$ of $O$, $V \subset U$, such that every orbit in $V$ is a cycle surrounding $O$.

The largest connected set $N_{O}$ covered with cycles surrounding $O$ is called central region. A center is said to be global if $N_{O} \equiv U$, that is, if every solution of (1) is periodic.

The two following definitions are due to Chavarriga, Giacomini, Giné and Llibre [14].

Definition 2.2. Let $H \in C^{k}(U, \mathbf{R}), k \geq 0$, be a (single-valued) function, non-constant on open sets. $H$ is said to be a strong first integral of system (1) if $H$ is constant on each solution of this system.

Here $k \geq 0$ means that $k=0,1,2, \ldots, \infty, \omega$. More precisely, $k=0$ means that $H$ is continuous, $k=1,2, \ldots, \infty$ means that $H$ is $C^{k}, k=\omega$ means that $H$ is analytic.

This definition of strong first integral is the usual definition of first integral which appears in most books on differential equations (see for instance Arnold [5], Farkas [43], Nemitskii and Stepanov [72], Sansone and Conti [92] and Sotomayor [99]). With this definition the linear differential system

$$
\dot{x}=x, \quad \dot{y}=y,
$$

\footnotetext{
${ }^{1}$ We would like to thank prof. R. Conti of the Universitá di Firenze and prof. A. Gasull of the Universitat Autònoma de Barcelona for having suggested some corrections in a previous version of this paper.
} 
defined on $\mathbf{R}^{2}$ has no strong first integrals. This is due to the fact that every strong first integral of system (2) must be a continuous function on $\mathbf{R}^{2}$ that takes a constant value on each straight line through the origin. Hence it has to be constant on all of $\mathbf{R}^{2}$, that contradicts the definition of strong first integral.

Definition 2.3. Let $\Sigma$ be the union of a family of orbits of system (1), and $H \in C^{k}(U \backslash \Sigma, \mathbf{R}), k \geq 0$, be a multi-valued function, non-constant on open sets. $H$ is said to be a weak first integral of system (1) if $H$ is constant on each solution of this system contained in $U \backslash \Sigma$.

With this definition the function $\arg (x, y)$ is a weak first integral of system (2). In this case $U \equiv \mathbf{R}^{2}, \Sigma=\{(0,0)\}$.

The use of multi-valued functions as first integrals has a long history. Thus, for instance, the first integrals computed by Darboux [39] for systems possessing sufficiently many algebraic solutions are in general of this kind (see also the book of Andronov, Vitt and Khaikin [4].)

We say that system (1) is a polynomial system if $X$ and $Y$ are polynomials with coefficients in $\mathbf{F}$, where $\mathbf{F}$ is either the real field $\mathbf{R}$ or the complex field $\mathbf{C}$; in this case, we write $X, Y \in \mathbf{F}[x, y]$. In both cases, we assume $t$ to be a real variable. We say that $m=\max \{\operatorname{deg} \mathrm{X}, \operatorname{deg} \mathrm{Y}\}$ is the degree of the polynomial system.

Definition 2.4. An invariant algebraic curve of system (1) is an algebraic curve $\mathrm{f}(\mathrm{x}, \mathrm{y})=0$ with $f \in \mathbf{F}[x, y]$, such that for some polynomial $K \in \mathbf{F}[x, y]$ we have

$$
\frac{\partial f}{\partial x} X+\frac{\partial f}{\partial y} Y=K f .
$$

We say that the curve $\mathrm{f}=0$ with $f \in \mathbf{F}[x, y]$ is an algebraic solution of system (1) if and only if it is an invariant algebraic curve and $\mathrm{f}$ is an irreducible polynomial over $\mathbf{F}[x, y]$.

We emphasize that $f$ can be a complex polynomial even in the case that $X, Y$ are real polynomials. If $f=0$ is a complex algebraic solution of a real system, then also its complex conjugate is a complex algebraic solution of the system. For instance, the system

$$
\dot{x}=-y, \quad \dot{y}=x,
$$

has the real algebraic solution $x^{2}+y^{2}=0$ and two complex algebraic solutions $x+i y=0$ and $x-i y=0$.

The polynomial $K$ is called the cofactor of the invariant algebraic curve $f=0$. We remark that if the polynomial system has degree $m$, then its cofactor has at most degree $m-1$. 
The work of Darboux contributed to show the link between the theory of polynomial systems and that of algebraic curves. Darboux showed how the first integrals of polynomial systems possessing sufficient algebraic solutions can be constructed. In particular, he proved that if a polynomial system of degree $m$ has at least $\frac{m(m+1)}{2}$ algebraic solutions, then it has a weak first integral.

Darboux's idea consists in looking for a first integral of the form $F=$ $\prod_{i=1}^{q} f_{i}^{\lambda_{i}}$ where $\lambda_{i} \in \mathbf{F}$ and $f_{i}=0$ are invariant algebraic curves of system (1). Such a first integral is called a Darboux first integral.

Some recent improvements to Darboux Theorem have been obtained by Chavarriga, Llibre and Sotomayor [21]. Essentially these improvements are based on the fact that the existence of a suitable number of singular points reduces the number $\frac{m(m+1)}{2}$ of algebraic solutions necessary for the integrability of the polynomial system.

Let $U$ be an open subset of $\mathbf{R}^{2}$ an let $R \in C^{k}(U, \mathbf{R})$ be an function which does not vanish on open subsets of $U$. The function $R$ is an integrating factor of system (1) on $U$ if

$$
\frac{\partial(R X)}{\partial x}=-\frac{\partial(R Y)}{\partial y} .
$$

The first integral $H$ associated to the integrating factor $R$ can be computed via the integral

$$
H(x, y)=\left(\int R(x, y) X(x, y) d y\right)+f(x)
$$

imposing the condition $\frac{\partial H}{\partial x}=-R Y$. Let $V$ be the reciprocal of an integrating factor. The function $V$ is namely a reciprocal integrating factor, and is a useful tool in the study of both integrable systems and non-integrable ones (see Chavarriga, Giacomini and Giné [13]).

In order to introduce next theorem we need some notation and definitions. Let $\mathbf{F}_{\mathbf{n}-\mathbf{1}}[x, y]$ be the set of polynomials $S(x, y)=\sum_{i+j=0}^{n-1} a_{i j} x^{i} y^{j}$ with its $\frac{n(n+1)}{2}$ coefficients in the field $\mathbf{F}$. We identify $\mathbf{F}_{\mathbf{n}-\mathbf{1}}[x, y]$ with the linear vector space $\mathbf{L}_{n-1}$, of dimension $\frac{n(n+1)}{2}$ by the isomorphism $S \rightarrow\left(a_{00}, a_{10}, a_{01}, \ldots, a_{n-1,0}, a_{n-2,1}, \ldots, a_{0, n-1}\right)$. We say that $p$ points $\left(x_{k}, y_{k}\right)$ with $k=1,2, \ldots, p$, are independent with respect to $\mathbf{L}_{n-1}$ if the intersection of the $p$ hyperplanes $\sum_{i+j=0}^{n-1} a_{i j} x_{k}^{i} y_{k}^{j}=0, k=1,2, \ldots, p$, in $\mathbf{L}_{n-1}$ is a linear subspace of dimension $\frac{n(n+1)}{2}-p$.

We remark that the maximum number of isolated singular points of sys- 
tem (1) is $m^{2}$ (by Bezout's Theorem), and that the maximum number of isolated singular points that are independent with respect to both components of system (1) is $\frac{m(m+1)}{2}$ and that $\frac{m(m+1)}{2}<m^{2}$ for $m \geq 2$.

A singular point $\left(x_{0}, y_{0}\right)$ of system (1) is called weak if the divergence $\operatorname{div}(X, Y)$, of system $(1)$ at $\left(x_{0}, y_{0}\right)$ is zero, that is

$$
(\operatorname{div}(X, Y))\left(x_{0}, y_{0}\right) \equiv\left[\frac{\partial X}{\partial x}+\frac{\partial Y}{\partial y}\right]\left(x_{0}, y_{0}\right)=0
$$

The main result of Darboux and the improvements of [21] are summarized in the following theorem.

TheOREM 2.1. Suppose that a polynomial system (1) of degree $m$ admits $q$ invariant algebraic curves $f_{i}=0$ with cofactors $K_{i}$ for $i=1,2, \ldots, q$, and $p$ independent singular points $\left(x_{k}, y_{k}\right)$ for $k=1,2, \ldots, p$, such that $f_{i}\left(x_{k}, y_{k}\right) \neq 0$. We have

(i) If there exist $\lambda_{i} \in \mathbf{F}$ not all zero such that $\sum_{i=1}^{q} \lambda_{i} K_{i}=0$, then $f_{1}^{\lambda_{1}} \ldots f_{q}^{\lambda_{q}}$ is a first integral, if $\mathbf{F}=\mathbf{C}$, or $\left|f_{1}\right|^{\lambda_{1}} \ldots\left|f_{q}\right|^{\lambda_{q}}$ is a first integral, if $\mathbf{F}=\mathbf{R}$.

(ii) If $q \geq \frac{m(m+1)}{2}+1-p$, then there exist $\lambda_{i} \in \mathbf{F}$ not all zero, such that $\sum_{i=1}^{q} \lambda_{i} K_{i}=0$

(iii) If $q \geq \frac{m(m+1)}{2}+2$ then there exist integer $\lambda_{i} \in \mathbf{F}$ such that $\sum_{i=1}^{q} \lambda_{i} K_{i}=$ 0. In particular the first integral given by (i) is rational, and consequently all solutions of the system are algebraic.

(iv) If there exist $\lambda_{i} \in \mathbf{F}$ not all zero, such that $\sum_{i=1}^{q} \lambda_{i} K_{i}=-\operatorname{div}(X, Y)$, then $R=f_{1}^{\lambda_{1}} \ldots f_{q}^{\lambda_{q}}$ is an integrating factor, if $\mathbf{F}=\mathbf{C}$, or $\left|f_{1}\right|^{\lambda_{1}} \ldots\left|f_{q}\right|^{\lambda_{q}}$ is an integrating factor, if $\mathbf{F}=\mathbf{R}$.

(v) If $q \geq \frac{m(m+1)}{2}-p>0$ and the $p$ independent singular points are weak, then there exist $\lambda_{i} \in \mathbf{F}$ not all zero, such that $\sum_{i=1}^{q} \lambda_{i} K_{i}=-\operatorname{div}(X, Y)$.

Let us consider a polynomial system (1) of degree $m$ with an isolated singular point which we place at the origin $O$. We consider the case where the linear part of system (1) at $O$ has pure imaginary eigenvalues $\lambda_{1,2}=$ $\pm i \omega, \omega \in \mathbf{R}, \omega \neq 0$. If we make a linear coordinate change and a time 
rescaling, we can write system (1) in the form

$$
\dot{x}=-y+\sum_{s=2}^{m} X_{s}(x, y), \quad \dot{y}=x+\sum_{s=2}^{m} Y_{s}(x, y) .
$$

with $X_{s}, Y_{s}$ homogeneous polynomials of degree $s, s=2,3, \ldots, m$. We have the following theorem due to Poincaré [79].

THEOREM 2.2. The origin of system (3) is a center if and only if, in an open neighbourhood of the origin, system (3) has a $C^{\omega}$ nonconstant strong first integral.

Poincaré's theorem has been generalized to $C^{k}$ systems, for $k=1, \ldots, \infty, \omega$, see Liapunov [60] and Mazzi and Sabatini [69]. We summarize their results in next theorem.

THEOREM 2.3. Let $X, Y$ be functions of class $C^{k}, k=1, \ldots, \infty, \omega$, in a neighbourhood $U$ of a singular point $O$ of system (1). Then $O$ is a center of system (1) if and only if system (1) has a strong first integral of class $C^{k}$, $k=1, \ldots, \infty, \omega$, in an open neighbourhood $V \subset U$ of $O$, with an isolated minimum at $O$.

Let $X, Y$ be analytic functions in a neighbourhood $U$ of a singular point $O$ of system (1), which we may place at the origin. Let $\lambda_{1,2}= \pm i \omega, \omega \in$ $\mathbf{R}, \omega \neq 0$ be the eigenvalues of the linear part at the origin $O$. We can make a linear coordinate change and a time rescaling, then we can write system (1) in the form

$$
\dot{x}=-y+\sum_{s=2}^{\infty} X_{s}(x, y), \quad \dot{y}=x+\sum_{s=2}^{\infty} Y_{s}(x, y),
$$

where the right hand side of (4) is a real power series, convergent in $U$, and $X_{s}, Y_{s}$ are homogeneous polynomials of degree $s, s=2,3, \ldots$.

The main analytic technique to determine whether $O$ is a center of system (4) or not was introduced by Poincaré [79] and developed by Liapunov [60]. It consists in looking for a formal power series of $x$ and $y$ of the form

$$
H(x, y)=\sum_{n=2}^{\infty} H_{n}(x, y), \quad H_{2}(x, y)=\frac{\left(x^{2}+y^{2}\right)}{2}
$$

where the $H_{n}(x, y)$ are homogeneous polynomials of degree $n, n=3,4, \ldots$, verifying

$$
\dot{H} \equiv\left[-y+\sum_{s=2}^{\infty} X_{s}(x, y)\right] \frac{\partial H}{\partial x}+\left[x+\sum_{s=2}^{\infty} Y_{s}(x, y)\right] \frac{\partial H}{\partial y}=
$$




$$
\sum_{k=1}^{\infty} V_{2 k}\left(x^{2}+y^{2}\right)^{k}
$$

with $V_{2 k}$ polynomials in the coefficients of system (4). The $V_{2 k}$ are called Liapunov constants. The first Liapunov constant different from zero is uniquely determined but the others are not. The sign of the first Liapunov constant different from zero gives us the stability of the origin: if it is negative the system has asymptotic stability for the positive times and if it is positive it has asymptotic stability for the negative times. As a consequence, all the Liapunov constants have to vanish, for $O$ to be a center of (4).

The converse statement is true, too. Poincaré [79] and Liapunov [60], showed that, when the Liapunov constants vanish, there exists a convergent series of type (5), satisfying (6). As a consequence, the origin is a center if and only if all the Liapunov constants are zero. In this case the convergent series is an analytic strong first integral of system (3) or (4) in a neighbourhood of the origin (see Poincaré [79] and Chazy [22]). In general, it is not always possible to express this first integral by means of elementary functions.

For polynomial systems, we have uniqueness of Liapunov constants in the sense of the following theorem due to Songling Shi [95]. Let us write

$$
X=-y+\sum_{i=2}^{n} \sum_{j=0}^{i} a_{i j} x^{i-j} y^{j}, Y=x+\sum_{i=2}^{n} \sum_{j=0}^{i} b_{i j} x^{i-j} y^{j}
$$

Theorem 2.4. Let $A$ be the ring $\mathbf{Q}\left[a_{i j}, b_{i j}\right], i=2,3, \ldots, n, j=0,1, \ldots, i$. Given a set of Liapunov constants $V_{i_{1}}, V_{i_{2}}, \ldots, V i_{p}$, let $J_{k-1}$ be the ideal of A generated by $V_{i_{1}}, V_{i_{2}}, \ldots, V_{i_{k-1}}, k \leq p$. If $V_{i_{1}}^{\prime}, V_{i_{2}}^{\prime}, \ldots, V^{\prime} i_{p}$ is another set of Liapunov constants, then $V_{i_{k}} \equiv V_{i_{k}}^{\prime} \bmod \left(J_{k-1}\right)$.

Let $J$ be the ideal of $A$ generated by all Liapunov constants. By Hilbert's basis theorem, $J$ is finitely generated, that is there exists $W_{1}, W_{2}, \ldots, W_{q}$ such that $J$ is generated by $W_{1}, W_{2}, \ldots, W_{q}$. Such a set of generators is called a basis. The vanishing of these generators implies the vanishing of all the Liapunov constants.

Several results concerning centers of polynomial sytems are based on Poincaré-Liapunov method. In the quadratic case, thanks to Dulac [42], Kapteyn [56], Frommer [44] and Bautin [9], it is known that there are exactly three independent Liapunov constants. It was Bautin [9] who determined the three Liapunov constants for the first time, and the first integrals were given by Lunkevich and Sibirskii [66]. From different points of view, $\mathrm{Li}$ Chengzhi [61], Lloyd [62], Chavarriga [11], Sibirskii [96] and Żolądek [114], 
each gave the three constants and characterized the different integrable cases, expressing the first integrals for each case.

In the cubic case with homogeneous nonlinearities, thanks to Sibirskii [97] and $\dot{Z}$ olạdek [115] it is known that there are five independent Liapunov constants. These were first given in cartesian coordinates by Sibirskii [97], and later by Lloyd [62]. The characterization of the integrable cases using polar coordinates, as well as the first integrals corresponding to each case were given by Chavarriga [11]. In the same work, he formulated a theorem that generalizes two integrable cases to polynomial systems of arbitrary degree.

Żoladek [116] gave partial results about the complete cubic case studying reversible cubic systems (systems with a center is said to be reversible if its orbits are symmetric with respect to a line passing through the center). Another significant result is the initial classification of Darboux integrals for cubic systems, by Sokulski [98]. These papers follow the idea of classifying the integrable systems according to the form of their first integral. Four groups appear (the ones with the Darboux Integral; those with the Darboux-Schwartz-Christoffel Integral; the systems that have the Darboux Hyperelliptic Integral and rationally reversible systems). From that point on, these problems are open. Nowadays, only some integrable cases are known for systems with fourth and fifth degree homogeneous nonlinearities, as given by Chavarriga and Giné [15, 16].

A special family of cubic systems, equivalent to a class of second order differential equations, is formed by the following systems

$$
\begin{aligned}
& \dot{x}=y, \\
& \dot{y}=-x+a_{1} x^{2}+a_{2} x y+a_{3} y^{2}+a_{4} x^{3}+a_{5} x^{2} y+a_{6} x y^{2}+a_{7} y^{3} .
\end{aligned}
$$

These systems were first studied by Kukles [58]. Kukles claimed to have proved a set of necessary and sufficient conditions for the origin to be a center of (8). Later, Jin and Wang [55] found an integrable example that does not verify the conditions set by Kukles. Lloyd and Pearson [63] found another different integrable case and they conjectured that there were no other possible integrable cases besides the known ones.

Finally, another family of cubic systems is the one with the form

$$
\dot{x}=-y+X_{2}(x, y)+X_{3}(x, y), \quad \dot{y}=x+Y_{2}(x, y)+Y_{3}(x, y),
$$

where $X_{s}(x, y)$ and $Y_{s}(x, y), s=2,3$, are homogeneous polynomials of degree $s$, verifying the condition $x Y_{3}(x, y)-y X_{3}(x, y) \equiv 0$ with $X_{3}^{2}+Y_{3}^{2} \not \equiv$ 0 . Such systems represent a specific case of cubic systems with degenerate infinity. This term was introduced by Sotomayor [100], and it owes its origin to the fact that, in Poincaré's compactification, every point at infinity is singular. These systems have been studied by Chen Guang-Qing and 
Liang Zhao-Jun [23], and, more recently, by Gasull and Prohens [47], who classified the quadratic systems with degenerate infinity.

Let $O$ be a center, and $V$ be a neighbourhood of $O$ covered with cycles surrounding $O$. We can define a function, the period function of $O$, by associating to every point $(x, y)$ of $V$ the minimal period of the cycle passing through $(x, y)$. A very special case is that of a constant period function, as in next definition.

Definition 2.5. A center $O$ of a system (1) is isochronous if the period of all integral curves in a punctured neighbourhood $V \backslash\{O\}$ of $O$ is constant.

The study of isochronous centers started when Huygens studied the cycloidal pendulum. This pendulum has isochronous oscillations, while the usual pendulum has oscillations of period increasing with the amplitude. The equations of the cycloidal pendulum are most easily written when the arc lenght $s$ is the independent variable

$$
\frac{d^{2} s}{d t^{2}}+k^{2} s=0
$$

where $k$ is a real constant. This is a special case of the following class of second order differential equations:

$$
\frac{d^{2} s}{d t^{2}}+g(s)=0 .
$$

Such equations arise in the study of conservative mechanical systems, that is mechanical systems that preserve the mechanical energy (= kinetic + potential energy). In the differential equations language, this is equivalent to the existence of a first integral of the form:

$$
H(s, \dot{s})=\frac{\dot{s}^{2}}{2}+G(s)
$$

where $G(s)=\int_{0}^{s} g(u) d u$. Urabe [101] and [102] studied the period function of (11), and proved a striking result: if we assume $g(s)$ to be an odd function, then the only differential equation of type (11) having an isochronous center at $O$ is (10). We shall deal in more detail with Urabe's results in the section concerned with hamiltonian systems.

Several classes of systems have been studied, in relation to the existence of isochronous centers. Among them, systems obtained from complex differential equations $\dot{z}=i P(z), z=x+i y$, were considered by Gregor [50], Hajek [51], [52], Lukashevich [65], Villarini [104], Mardešić, Rousseau and Toni [68], Christopher and Devlin [28], Sabatini [87]; quadratic systems, by Loud [64], Chicone and Jacobs [27], Sabatini [85]; cubic systems with homogeneous nonlinearities by Pleshkan [76], Pyzhkova and Cherenkova [80], 
Collins [34], Gasull, Guillamón and Mañosa [45], Mazzi and Sabatini [70], Rousseau and Toni [82]; Kukles systems (8) by Christopher and Devlin [28], Rousseau and Toni [83], Mardešić, Rousseau and Toni [68]; cubic reversible systems with constant angular speed, by Mardešić, Rousseau and Toni [68], Collins [34], Mazzi and Sabatini [70], Christopher and Devlin [28], Chavarriga and García [12]; cubic reversible systems, by Chavarriga and García [12]; cubic systems with degenerate infinity (9), by Christopher, Devlin, Lloyd, Pearson and Yasmin [30], Chavarriga, Giné and García [18]; quartic and quintic systems with homogeneous nonlinearities by Chavarriga, Giné and García [19]; systems with a fractional vector field by Pleshkan [77].

Centers with a nonconstant period function received a significant attention, too. We are also interested in such results, because they can be considered as negative results about isochronicity. Anyway, we shall not go into details about them. We only quote some papers in which centers with non-constant period functions are studied, since they are naturally related to some of the isochronicity cases considered in the present paper. Thus, for instance Waldvogel [111], Hsu [53], Rothe [81], Chicone and Dumortier [26], Coppel and Gavrilov [38] considered quadratic systems; Waldvogel [111], Schaaf [93], Chicone [25], Collins [33], Gasull, Guillamon, Mañosa and Mañosas [46] hamiltonian systems; Urabe [102], Opial [75] and Obi [73] second order conservative differential equations; Cima, Gasull, Mañosa and Mañosas [31] and Sabatini [90] Liénard differential equations.

Finally, we observe that there exist differential systems that have both isochronous centers and non-isochronous ones. The coexistence of isochronous centers and nonisochronous centers has been studied by Devlin [40].

\section{LINEARIZATIONS}

Given the plane differential system (1), we say that (1) is $C^{k}$-linearizable at a point $O$ if there exists a local $C^{k}$-diffeomorphism $\Phi$ defined in a neighbourhood of $O$ that transforms (1) into a linear differential system (see Irwin [54]). By the local rectification theorem, every system can be linearized at a nonsingular point. On the other hand, this is not true at singular points, since diffeomorphisms preserve the index, and singular points of linear systems can only have index 1,0 or -1 . Moreover, even in the case of singular points with the same index, in general there are no diffeomorphisms taking locally one system into the other. In fact, diffeomorphisms preserve cycles' periods, hence two centres with different period functions cannot be transformed into each other by any diffeomorphism. Centers of linear systems are isochronous, so that a necessary condition for a system with a center at $O$ to be linearizable is that $O$ be isochronous. For analytic systems, such a condition is also sufficient for the linearizability (see theorem 3.3). 
Let us consider an analytic system with a center at $\mathrm{O}$. We recall that a center is said to be nondegenerate if the determinant of the linear part at $O$ is different from zero. Every system with a nondegenerate center at $\mathrm{O}$ can be reduced, by a linear transformation and a time rescaling, into the form (4). Next theorem is due to Poincaré.

THEOREM 3.1. For an analytic system (4) with a nondegenerate center there exists an analytic change of coordinates of the form $\chi=x+o(|(x, y)|)$, $\eta=y+o(|(x, y)|)$ and an analytic function $\Psi$ such that the coordinate change transforms the system (4) into a system of the form

$$
\dot{\chi}=-\eta\left(1+\Psi\left(\chi^{2}+\eta^{2}\right)\right), \quad \dot{\eta}=\chi\left(1+\Psi\left(\chi^{2}+\eta^{2}\right)\right) .
$$

Without loss of generality, we can assume that $\Psi(0,0)=0$.

Following Mardešić, Rousseau and Toni [68], we observe that, by theorem 2.3 , if system $(4)$ has a center at $\mathrm{O}$, then there exists a strong first integral of the form $F(x, y)=x^{2}+y^{2}+o\left(|(x, y)|^{2}\right)$. Since all terms of degree higher than 2 are divisible either by $x^{2}$ or by $y^{2}$, the first integral can be written in the form $F(x, y)=x^{2}(1+f(x, y))+y^{2}(1+g(x, y))$. The analytic change of coordinates $\chi=x \sqrt{1+f(x, y)}, \eta=y \sqrt{1+g(x, y)}$ transforms system (4) into

$$
\dot{\chi}=-\eta(1+G(\chi, \eta)), \quad \dot{\eta}=\chi(1+G(\chi, \eta)),
$$

where $G(\chi, \eta)$ is an analytic function. As a consequence, we have the following theorem.

THEOREM 3.2. The analytic system (4) has an isochronous center at $O$ if and only if

$$
\int_{0}^{2 \pi} \frac{d \varphi}{1+G(r \cos \varphi, r \sin \varphi)}=2 \pi
$$

In the special case of theorem 3.1, the condition (15) implies $\Psi\left(\chi^{2}+\eta^{2}\right) \equiv$ const. Hence, the following theorem holds.

THEOREM 3.3. A center of an analytic system (4) is isochronous if and only if there exists an analytic change of coordinates of the form

$$
\chi=x+o(|(x, y)|), \quad \eta=y+o(|(x, y)|),
$$

reducing the system to the linear isochronous system

$$
\dot{\chi}=-k \eta, \quad \dot{\eta}=k \chi
$$


where $k$ is a non-zero real constant.

For a discussion of theorems 3.1, 3.2 and 3.3 and their applicability, see Mardešić, Rousseau and Toni [68].

From theorem 3.3, it is evident that a necessary condition for an analytic center $O$ to be isochronous is that $O$ is a nondegenerate singular point. Such a condition has been extended to $C^{\infty}$ vector fields in Villarini [104].

A special role in the study of polynomial systems is played by Darboux linearizations. Next definitions will be given in complex notation. Let us set $z=x+i y, \bar{z}=x-i y$. If we assume $X(x, y)$ and $Y(x, y)$ to be polynomials, and $O$ to be a nondegenerate center, then, after a linear change of variables and a rescaling of time, system (3) can be written as

$$
\dot{z}=i z+\sum_{i+j=2}^{n} \alpha_{i j} z^{i} \bar{z}^{j}, \quad \alpha_{i j} \in \mathbf{C} .
$$

A Darboux function is a function $\zeta(z)$ of the form:

$$
\zeta(z)=\prod_{j=0}^{h} F_{j}^{\alpha_{j}}(z), \quad \alpha_{j} \in \mathbf{C},
$$

where the function $F_{j}(z)$ are either complex polynomials, or exponentials of polynomials.

A Darboux linearization is a Darboux function that is a local diffeomorphism at $\mathrm{O}$, and takes system (17) into the system:

$$
\dot{z}=i z .
$$

The above system is just the linear system

$$
\dot{x}=-y, \quad \dot{y}=x,
$$

written in complex notation.

If a system is Darboux linearizable, then it is Darboux integrable, that is, it has a Darboux function as a first integral.

In Mardešić, Rousseau and Toni [68] and Mardešić, Moser-Jauslin and Rousseau [67], the existence of Darboux linearizations for several classes of polynomial systems is discussed.

\section{COMPUTATION OF ISOCHRONOUS CONSTANTS}

In the study of isochronous centers it is often convenient to use polar coordinates. In Lemma 4.1 we give the expression of an analytic system (4) in polar coordinates (see [11]). 
Lemma 4.1. In polar coordinates $x=r \cos (\varphi), y=r \sin (\varphi)$ we can write system (4) as follows

$$
\dot{r}=\sum_{s=2}^{\infty} P_{s}(\varphi) r^{s}, \quad \dot{\varphi}=1+\sum_{s=2}^{\infty} Q_{s}(\varphi) r^{s-1}
$$

where $P_{i}(\varphi)$ and $Q_{i}(\varphi)$ are trigonometric polynomials of the form

$$
\begin{aligned}
P_{s}(\varphi) & =R_{s+1}^{s} \cos \left((s+1) \varphi+\varphi_{s+1}^{s}\right)+R_{s-1}^{s} \cos \left((s-1) \varphi+\varphi_{s-1}^{s}\right)+\ldots+ \\
& +\left\{\begin{array}{cl}
R_{1}^{s} \cos \left(\varphi+\varphi_{1}^{s}\right) & \text { if } s \text { is even; } \\
R_{0}^{s} & \text { if } s \text { is odd; }
\end{array}\right. \\
Q_{s}(\varphi) & =-R_{s+1}^{s} \sin \left((s+1) \varphi+\varphi_{s+1}^{s}\right)+r_{s-1}^{s} \sin \left((s-1) \varphi+\bar{\varphi}_{s-1}^{s}\right)+\ldots+ \\
& +\left\{\begin{array}{cl}
r_{1}^{s} \sin \left(\varphi+\bar{\varphi}_{1}^{s}\right) & \text { if } s \text { is even; } \\
r_{0}^{s} & \text { if } s \text { is odd; }
\end{array}\right.
\end{aligned}
$$

with $s=1,2, \ldots$ and where $R_{j}^{s}, r_{j}^{s}, \varphi_{j}^{s}$ and $\bar{\varphi}_{j}^{s}$ are arbitrary coefficients.

Theorem 4.1 gives the form of hamiltonian systems and reversible systems in polar coordinates (see [11]).

THEOREM 4.1. System (21) has a center at the origin in the following two cases:

(i) (Hamiltonian) If $(s+1) P_{s}+\frac{d Q_{s}}{d \varphi} \equiv 0$ for all $s=1,2, \ldots$

(ii)(Reversible) If $P_{s}(\varphi)$ and $Q_{s}(\varphi)$ are of the form

$$
\begin{aligned}
P_{s}(\varphi) & =R_{s+1}^{s} \sin (s+1) \omega+R_{s-1}^{s} \sin (s-1) \omega+\ldots+ \\
& + \begin{cases}R_{1}^{s} \sin \omega, & \text { if } s \text { is even; } \\
R_{2}^{s} \sin 2 \omega & \text { if } s \text { is odd; }\end{cases} \\
Q_{s}(\varphi) & =R_{s+1}^{s} \cos (s+1) \omega+r_{s-1}^{s} \cos (s-1) \omega+\ldots+ \\
& +\left\{\begin{array}{cc}
r_{1}^{s} \cos \omega, & \text { if } s \text { is even; } \\
r_{2}^{s} \cos 2 \omega+r_{0}, & \text { if } s \text { is odd; }
\end{array}\right.
\end{aligned}
$$

for all $s=2,3, \ldots$ where $\omega=\varphi+\varphi_{0}$, and $\varphi_{0}, R_{j}^{s} r_{j}^{s}$ are arbitrary coefficients.

Lemma 4.1 and Theorem 4.1 are proved in Chavarriga [11].

Theorem 3.3 is the basis for the method that we describe in this section, that is similar to Poincaré-Liapunov method for the identification of centers.

Let the origin be an isochronous center of system (4). By theorem 3.3, we know that for system (4) there exists an analytic change of coordinates 
$\chi=x+o(|(x, y)|), \eta=y+o(|(x, y)|)$ such that $\ddot{\chi}+\chi=0$ and $\ddot{\eta}+\eta=0$, where we have taken $k=1$ making a rescaling of the time. We can use polar coordinates $x=r \cos \varphi, y=r \sin \varphi$. Let $\chi=H(r, \varphi)$ be a power series of the form

$$
H(r, \varphi)=\sum_{i=1}^{\infty} H_{i}(\varphi) r^{i},
$$

where $H_{1}(\varphi)=\cos \varphi$ and $H_{i}(\varphi), i=1,2, \ldots$, are homogeneous trigonometric polynomials of degree $i$.

Let us call $\ddot{H}$ the second derivative of $H$ along the solutions of (12). Then, let us impose that $\ddot{H}+H=0$. Thus we obtain a system of recursive differential equations given by

$$
\begin{aligned}
& H_{n+1}^{\prime \prime}+H_{n+1}+\sum_{\substack{k=1 \\
i+j+k=n+3}}^{n}\left[Q_{i} Q_{j} H_{k}^{\prime \prime}+\left((j+2 k-1) P_{i} Q_{j}+Q_{i} Q_{j}^{\prime}\right) H_{k}^{\prime}+\right. \\
& \left.k\left[P_{i}^{\prime} Q_{j}+\frac{k+n+1}{2} P_{i} P_{j}\right] H_{k}\right]=\left\{\begin{array}{cc}
\alpha_{n+1} \cos \varphi+\beta_{n+1} \sin \varphi, & \text { if } n \text { is odd } \\
0, & \text { if } n \text { is even; }
\end{array}\right.
\end{aligned}
$$

where $n=1,2, \ldots,{ }^{\prime}=\frac{d}{d \varphi}, P_{1} \equiv 0, Q_{1} \equiv 1$ and $H_{1}(\varphi)=\cos \varphi$, with $\alpha_{n+1}$ and $\beta_{n+1}$ are real numbers called isochronous constants.

The isochronous constants are polynomials in the parameters of system (4). The vanishing of all isochronous constants is a necessary condition for the isochronicity of the origin. Moreover, if system (4) is polynomial then by the Hilbert's basis theorem exists $q \in \mathbb{N}$ such that the ideal generate by the isochronous constants is finitely generated by $\alpha_{j}, \beta_{j}, \alpha_{j_{2}}, \beta_{j_{2}}, \ldots, \alpha_{j_{q}}, \beta_{j_{q}}$. Unfortunately, in general the number $q$ is a priori unknown, so that the applicability of this method meets the same restrictions of Poincaré-Liapunov method for the identification of centers (see the previous section). In general, proving that one of the period constants is different from zero shows that the center is not isochronous, while proving that finitely many period constants are zero proves the isochronicity only under some additional conditions. For a discussion of this method see, for instance, Cima, Gasull, Mañosa and Mañosas [31].

\section{COMMUTING SYSTEMS}

In this section we denote by $z=(x, y)$ a point of the plane. Let us consider a couple of differential systems defined on an open, connected subset $U$ of the plane

$$
\dot{z}=V(z), \quad V \equiv\left(v_{1}, v_{2}\right) \in C^{k}\left(U, \mathbf{R}^{2}\right), \quad k \geq 2,
$$




$$
\dot{z}=W(z), \quad W \equiv\left(w_{1}, w_{2}\right) \in C^{k}\left(U, \mathbf{R}^{2}\right), \quad k \geq 2 .
$$

We denote by $\phi(t, z)$ (resp. $\psi(s, z))$ the solution of $(24)((25))$, such that $\phi(0, z)=z(\psi(0, z)=z)$.

Let $T, S$ be positive real numbers, and $P=[0, T] \times[0, S]$ be a rectangle, that will be called a parametric rectangle.

Definition 5.1. We say that the local flows $\phi(t, z)$ and $\psi(s, z)$ commute if, for every parametric rectangle $P$ such that both $\phi(t, \psi(s, z))$ and $\psi(s, \phi(t, z))$ exist whenever $(t, s) \in P$, one has

$$
\phi(t, \psi(s, z))=\psi(s, \phi(t, z)) .
$$

By a classical result, two local flows commute if and only if the Lie brackets $[V, W]$ of $V$ and $W$ vanish identically on $U$ (see Arno'ld [6], Olver $[74])$ :

$$
\begin{aligned}
& {[V, W]_{1}=\left(v_{1} \frac{\partial w_{1}}{\partial x}-w_{1} \frac{\partial v_{1}}{\partial x}\right)+\left(v_{2} \frac{\partial w_{1}}{\partial y}-w_{2} \frac{\partial v_{1}}{\partial y}\right) \equiv 0} \\
& {[V, W]_{2}=\left(v_{1} \frac{\partial w_{2}}{\partial x}-w_{1} \frac{\partial v_{2}}{\partial x}\right)+\left(v_{2} \frac{\partial w_{2}}{\partial y}-w_{2} \frac{\partial v_{2}}{\partial y}\right) \equiv 0 .}
\end{aligned}
$$

In this case we say that $V$ and $W$ commute, or that $W$ is a commutator of $V$. When dealing with commutators, we always assume that the following conditions hold. We recall that $V$ and $W$ are said to be transversal at a point $z$ if $v_{1}(z) w_{2}(z)-v_{2}(z) w_{1}(z) \neq 0$.

(i) $\left(S_{V}\right)$ and $\left(S_{W}\right)$ have isolated critical points in $U$;

(ii) $V(z)=0$ if and only if $W(z)=0$;

(iii) if $z \in U$, and $V(z) \neq 0$, then $V(z)$ and $W(z)$ are transversal;

(iv) $[V, W] \equiv 0$ on $\mathrm{U}$.

The equations (27) assume a very special form if $W \equiv\left(v_{2},-v_{1}\right)$. In this case, we write $V_{\perp}$ for $\mathrm{W}$.

$$
\begin{aligned}
& {\left[V, V_{\perp}\right]_{1}=v_{1}\left(\frac{\partial v_{2}}{\partial x}+\frac{\partial v_{1}}{\partial y}\right)+v_{2}\left(\frac{\partial v_{2}}{\partial y}-\frac{\partial v_{1}}{\partial x}\right)} \\
& {\left[V, V_{\perp}\right]_{2}=v_{1}\left(\frac{\partial v_{2}}{\partial y}-\frac{\partial v_{1}}{\partial x}\right)-v_{2}\left(\frac{\partial v_{2}}{\partial x}+\frac{\partial v_{1}}{\partial y}\right) .}
\end{aligned}
$$

It is evident that if $v_{1}$ and $v_{2}$ satisfy Cauchy-Riemann equations, then the equations (28) hold. This simple observation led Villarini [104] to give a very elegant geometric proof of Gregor's results [50] about complex systems (see next section). Sabatini $[84,86]$ later oberved that Villarini's proof can be extended to couples of transversal commuting vector fields, not necessarily orthogonal to each other. In [86] it was proved that commutativity can be used to characterize isochronous centers, as stated in next theorem. 
THEOREM 5.1. Let $O$ be a center of system (1). Then $O$ is an isochronous center if and only if there exists a vector field $W$ defined in a neighbourhood of $O$, that is transversal to $V$ at nonsingular points, and commutes with $V$.

Villarini [105] extended this result showing that $V$ has always a commutator with nondegenerate linear part. A different proof, for analytic systems, can be found in Algaba, Freire and Gamero [1]. Both constructions in Sabatini [86] and Villarini [105] show that the commutator exists on all of the central region.

In some cases, looking for a commutator is easier than looking for linearizations. For instance, quadratic systems admit polynomial commutators of degree $\leq 4$ (see Sabatini [85]), while polynomial linearizations of quadratic systems are not known. The simplest known linearizations of quadratic systems are rational (see Mardešić, Rousseau and Toni [68]). On the other hand, it is not true that every polynomial system with an isochronous center has a polynomial commutator. Let us consider Devlin's example [40]:

$$
\dot{x}=-y-x^{4}+4 x^{2} y^{2}+y^{4} \quad \dot{y}=x-4 x^{3} y .
$$

This is a polynomial system having an isochronous center and two nonisochronous centers. If a polynomial commutator existed, then it would be defined on all of the plane, and the commutativity conditions would be satisfied at every point of the plane. Then every center should be isochronous, contradicting the existence of a non-isochronous center. A discussion and some results about the existence of polynomial commutators for polynomial systems can be found in Volokitin and Ivanov [106].

Sometimes polynomial systems do not satisfy all conditions (i), . , (iv). In particular, it is possible for one of the two vector fields to have nonisolated singularities. In this case we can reduce to the complement of the set of non-isolated singularities, since commuting flows preserve transversality. Let us call reachable set $R(z)$ the set of points that can be connected to $z$ by means of finitely many arcs of orbits of $V$ and $W$. In [87] it was showed that if $V$ and $W$ are transversal at $z \in \mathbf{R}^{2}$, then they are transversal at every point of $R(z)$. This is a consequence of the following property of couples of commuting systems. Let us write $A=v_{1} w_{2}-v_{2} w_{1}$. $A$ vanishes at $z$ if and only if $V$ and $W$ are non-transversal at $z$. We have

$$
\partial_{V} A=A \operatorname{div} V, \quad \partial_{W} A=A \operatorname{div} W,
$$

so that $A$ vanishes at $z$ if and only if it vanishes on all the $V$-orbit and the $W$-orbit through $z$. As a consequence, if $V$ and $W$ are transversal at a point of $N_{O}$, they are transversal on all of $N_{O}$.

The function $A$ has also an important role in the integrability of both (24) and (25). Let us observe that a function $B$ is an integrating factor of 
both $V$ and $W$ if and only if:

$$
\partial_{V} B=-B \operatorname{div} V, \quad \partial_{W} B=-B \operatorname{div} W .
$$

Comparing the above equations leads to the following conclusion (see Sabatini [89] and Chavarriga, Giacomini and Giné [13]).

Theorem 5.2. Let $[V, W] \equiv O$. Then $v_{1} w_{2}-v_{2} w_{1}$ is a reciprocal integrating factor of both $V$ and $W$.

By theorem 5.2, if one knows a commutator $W$ of $V$, then it is possible to find a first integral of both (24) and (25) by integrating a suitable differential form. Anyway, in general it is not possible to find strong first integrals of both systems. For instance, if (24) has a center $O$ and (25) an asymptotically stable point at $O$, in a neighbourhood of $O$ one finds a strong first integral for (24) and a weak first integral for (25). On the other hand, in a neighbourhood of a regular point $z$, one finds strong first integrals of both systems, and can even rectify locally $V$ and $W$ (see Sabatini [89]).

Another noteworthy feature of commutators is the possibility to prove the existence of centers. We say that $\left\{V(\theta) \in C^{k}\left(U, R^{2}\right), \theta \in[0,2 \pi)\right\}$ is a complete family of commuting vector fields on $U$, if $V(\theta)$ is a complete family of rotated vector fields (see Duff [41] for the definition), and $\forall \theta_{1}, \theta_{2} \in$ $[0,2 \pi)$, one has $\left[V\left(\theta_{1}\right), V\left(\theta_{2}\right)\right] \equiv 0$ on $U$. If one of the vector fields in a complete family has a point of index 1 at $O$ then every other vector field has the same index, with the possibility of limit cycle bifurcations as the parameter $\theta$ varies. If the family consists of commuting vector fields, no such bifurcations can occur, so that the following theorem holds.

THEOREM 5.3. Let $V(\theta)$ be a complete family of commuting vector fields. Then there exists a parameter $\theta^{*} \in[0, \pi)$ such that $O$ is a center for both $V\left(\theta^{*}\right)$ and $V\left(\theta^{*}+\pi\right)$ and for no other values of $\theta$.

The following corollary has been used by Mazzi and Sabatini [70] to prove the integrability of a class of systems.

Corollary 5.1. Let $p(x, y), q(x, y), r(x, y), s(x, y) \in C^{2}(U, \mathbf{R})$. If the system

$$
\dot{x}=-y+p(x, y), \quad \dot{y}=x+q(x, y),
$$

commutes with the system

$$
\dot{x}=x+r(x, y), \quad \dot{y}=y+s(x, y),
$$

where $p(x, y), q(x, y), r(x, y), s(x, y)=o(|(x, y)|)$, then the origin is an isochronous center of (31). 
There is a natural relationship between commutators and linearizations. Let us assume that there exists a local diffeomorphism $\Phi$ taking (5) into the linear system:

$$
\dot{x}=-y \quad \dot{y}=x .
$$

Then system (34) has a linear commutator:

$$
\dot{x}=x \quad \dot{y}=y .
$$

The transformation $\Phi^{-1}$ takes (35) into a system that commutes with (5). $\Phi$ linearizes both and its commutator. It is natural to ask whether the converse statement is true. Given a couple of commuting vector fields, is it possible to find a transformation that linearizes both systems? For analytic systems, the answer is in the following theorem by Bambusi, Cicogna, Gaeta and Marmo [7].

THEOREM 5.4. An analytic system of the form (5) is analytically linearizable if and only if it admits an analytic commutator of the form (33). Moreover, there exists an analytic transformation that linearizes both (5) and (33).

For non-analytic systems, a partial result in the same direction has been given in Mazzi and Sabatini [70] and Villarini [105].

THeOREM 5.5. Under the hypotheses of corollary 5.1, if a local diffeomorphism $\Phi$ linearizes system (32), then it linearizes system (31) as well.

\section{COMPLEX SYSTEMS}

The first general class of plane systems for which isochronicity was proved is that of the so-called complex systems. Let us consider the following differential equation, where the unknown function is $t \mapsto z(t), t \in R$, $z \in \mathbf{C}$ :

$$
\dot{z}=F(z) .
$$

This complex differential equation can be written as a differential system in the real plane of the form (1), where $X=\operatorname{Re} F$ and $Y=\operatorname{Im} F$.

The first results about the isochronicity of complex systems was probably given by Gregor [50], whose results can be found summarized in Hajek [51]. We report his results in next theorem.

Theorem 6.1. Let us consider system (1). Let $F=X+i Y$ be meromorphic. Then: 
(i) every pole of $F$ is a saddle point of system (1);

(ii) a multiple zero $O$ of $F$ is a node if $\operatorname{Res}\left(\frac{1}{F}, O\right)=0$;

(iii) a simple zero of $F$ is either a node or a focus or a center;

(iv) a simple zero $O$ of $F$ is a center of system (1) if and only if $F^{\prime}(O)$ is purely imaginary. In this case $O$ is an isochronous center. The common period of the cycles $C$ surrounding $O$ is:

$$
T=\frac{2 \pi i}{F^{\prime}(O)} i n d_{C} O
$$

where ind $_{C} O$ is the index of the cycle $C$ with respect to $O$.

Independently, Lukashevich [65] obtained some of Gregor's results. He considered only the case of an holomorphic $F$, showing that in this case system (1) can be analytically linearized in a neighbourhood of a simple singular point $O$. He also proved that system (1) cannot have limit cycles.

Hajek [51] and [52] extended Gregor's results analyzing the global qualitative behaviour of the solutions of system (1). He gave a different proof of the absence of limit cycles of system (1).

Recently Villarini [104] developped a geometric approach to the study of complex systems. He showed that if $F$ is holomorphic, then the solutions of system (1) and those ones of its orthogonal system:

$$
\dot{x}=-Y(x, y), \quad \dot{y}=X(x, y),
$$

commute. This allowed him to give one-line proofs of the isochronicity of centers and of the absence of limit cycles.

By theorem 3.3, if $X+i Y$ is holomorphic, then system (1) can be analytically linearized. The explicit form of the linearization, and an expression of the first integral, were given in Mardešić, Rousseau and Toni [68] for polynomial systems. We report them in next table. We denote by $z_{j}, j=1, \ldots, r$ the zeroes of $F(z)$. For other results about complex systems, see Christopher and Devlin [28].

\section{HAMILTONIAN SYSTEMS}

Let us consider $H \in C^{k}(U, \mathbf{R}), k \geq 2, U$ open connected subset of $\mathbf{R}^{2}$ containing the origin $\mathrm{O}$. The differential system

$$
\dot{x}=\frac{\partial H}{\partial y}, \quad \dot{y}=-\frac{\partial H}{\partial x},
$$


TABLE 1.

Complex Systems.

\section{Complex System}

Cartesian coordinates:

$$
\dot{x}=X(x, y),
$$$$
\dot{y}=Y(x, y) \text {. }
$$

Under the conditions:

$$
\begin{gathered}
\frac{\partial X}{\partial x}=\frac{\partial Y}{\partial y}, \\
\frac{\partial X}{\partial y}=-\frac{\partial Y}{\partial x} .
\end{gathered}
$$

Transversal commuting system:

$$
\begin{gathered}
\dot{x}=-X(x, y), \\
\dot{y}=Y(x, y) .
\end{gathered}
$$

Linearization (in complex notation): $\zeta(z)=z \prod_{j=1}^{r}\left(z-z_{j}\right)^{\operatorname{Res}\left(\frac{1}{F}, z_{j}\right)} e^{Q(z)}$, where:

$Q(z)=\int\left(\frac{1}{F}-\sum_{j=1}^{r} \frac{\operatorname{Res}\left(\frac{1}{F}, z_{j}\right)}{z-z_{j}}-\frac{1}{z}\right) d z$ Strong first integral:

$$
\zeta^{-1}(z \bar{z})
$$

Reciprocal integrating factor:

$$
V=\left(z \bar{z} \prod_{j=1}^{r}\left(z-z_{j}\right)\left(\bar{z}-\overline{z_{j}}\right)\right) .
$$


is known as the hamiltonian system associated to $H$. A special case is that of second order differential equations of type (11) arising from conservative mechanical systems. In this case one has

$$
H(x, y)=\frac{y^{2}}{2}+G(x)
$$

where $G(x)=\int_{0}^{x} g(s) d s$, so that the plane system, equivalent to the differential equation (11), is

$$
\dot{x}=y, \quad \dot{y}=-g(x) \text {. }
$$

If $x g(x)>0$ for small $x \neq 0$, then $O$ is a centre of (39). The main result concerning system (39) is due to Urabe [101] (see also Opial [75], Levin and Shatz [59]). If $x g(x)>0$ for small $x \neq 0$, the transformation

$$
X=\mu(x)=\operatorname{sgn}(x) \sqrt{2 G(x)},
$$

admits an inverse in a neighbourhood of 0:

$$
x=\mu^{-1}(X) .
$$

The function $\mu^{-1}(X)$ plays a key role in Urabe's characterization of isochronous centers of (39).

THEOREM 7.1. System (39) has an isochronous center at the origin with constant period $\omega$ if and only if there exist scalar functions $\sigma(X), \tau(X)$ defined in a neighbourhood of 0 , such that:

(i) $\sigma$ is odd, continuous, $X \sigma(X)$ is of class $C^{1}, \sigma(0)=0$;

(ii) $\tau$ is even, continuous, $X \tau(X)$ is of class $C^{1}, \tau(0)=0$;

(iii)

$$
g\left(\mu^{-1}(X)\right)=\frac{2 \pi}{\omega} \frac{X}{1+\sigma(X)+\tau(X)} ;
$$

(iv) for every $r>0, \quad \int_{0}^{\pi / 2} \tau(r \cos \varphi) d \varphi=0$.

The most relevant consequence of theorem 7.1 is the following corollary (see Urabe [101]).

COROLlary 7.1. Let $g$ be an odd analytic function. Then system (39) has an isochronous center at the origin if and only if $g(x)=k^{2} x$, for some $k \in \mathbf{R}, k \neq 0$. 
The following theorem was proved independently by Amel'kin [3] and Chicone and Jacobs [27].

THeORem 7.2. Let $g$ be a polynomial. Then system (39) has an isochronous center at the origin if and only if $g(x)=k^{2} x$, for some $k \in \mathbf{R}$, $k \neq 0$.

Both corollary 7.1 and theorem 7.2 can be proved following a different approach, developped recently by Cima, Mañosas and Villadelprat [32]. Let us define a strict involution as an analytic function $\gamma: \mathbf{R} \mapsto \mathbf{R}$ such that $\gamma(0)=0, \gamma(\gamma(x)) \equiv x, \gamma(x) \not \equiv x$.

THEOREM 7.3. Let $g$ be analytic. Then the origin is an isochronous center of period $\omega$ of (39) if and only if there exists a strict involution $\gamma$ such that

$$
G(x)=\frac{\pi^{2}}{2 \omega^{2}}(x-\gamma(x))^{2}
$$

for all $x \in \mathbf{R}$.

Theorem 7.3 gives a simple procedure to construct systems of type (39) having an isochronous center, with $g(x)$ analytic, non-odd and non-polynomial.

If $g(x)$ is nonlinear, a great variety of situations can occur. In fact, Urabe [102] proved that for every continuous, positive scalar function $\lambda: \mathbf{R}^{+} \mapsto \mathbf{R}$ there exists a $g_{\lambda}(x): \mathbf{R}^{+} \mapsto \mathbf{R}$ such that the differential equation

$$
\ddot{x}+g_{\lambda}(x)=0
$$

has a center with period function given by $\lambda$ (see also [113]).

An analogous to Theorem 7.2 does not hold for plane hamiltonian systems: there exist nonlinear polynomial hamiltonian systems with isochronous centers. A method to produce them consists in transforming a linear center into a nonlinear one by means of a nonlinear polynomial canonical transformation having a polynomial inverse. Such transformations are a special subclass of a wider family of maps, that are the object of a celebrated conjecture, known as the Jacobian Conjecture.

Jacobian Conjecture Let $\Phi: \mathbf{C}^{n} \rightarrow \mathbf{C}^{n}$ be a polynomial map with constant non-vanishing jacobian determinant. Then $\Phi$ is a global diffeomorphism and its inverse is a polynomial map.

Such a problem was studied in several different settings (see Bass, Connell and Wright [8]). It is known that it is equivalent to solve it in $\mathbf{R}^{n}$ or in $\mathbf{C}^{n}$, and several partial results have been proved, but the conjecture is still unproved $\forall n \geq 2$. The Jacobian Conjecture motivates the following definition. 
Definition 7.1. A map $\Phi(x, y)=(P(x, y), Q(x, y)), \Phi \in C^{1}\left(U, \mathbf{R}^{2}\right), \mathrm{U}$ open connected subset of the plane, is said to be a jacobian map if

$$
J_{\Phi}:=\operatorname{det}\left(\begin{array}{cc}
\frac{\partial P}{\partial x} & \frac{\partial P}{\partial y} \\
\frac{\partial Q}{\partial x} & \frac{\partial Q}{\partial y}
\end{array}\right) \equiv c \neq 0, \quad c \in \mathbf{R}
$$

There exists a natural relationship between the study of the Jacobian Conjecture and the study of hamiltonian isochronous centers. Before describing it, we report a result by Vorob'ev [109], concerned with general systems, not necessarily polynomial (see also $[107,108,110]$ ).

Theorem 7.4. Let $\Phi(x, y)=(P(x, y), Q(x, y)), \Phi \in C^{1}\left(U, \mathbf{R}^{2}\right)$, be a map defined on $U$, open connected subset of the plane, $P(0,0)=Q(0,0)=$ $0, J_{\Phi}(0,0)=1, J_{\Phi}(x, y)>0$ for all $(x, y) \in U$. Then the system

$$
\dot{x}=-\frac{1}{J_{\Phi}} \frac{\partial\left(P^{2}+Q^{2}\right)}{\partial y} \quad \dot{y}=\frac{1}{J_{\Phi}} \frac{\partial\left(P^{2}+Q^{2}\right)}{\partial x}
$$

has an isochronous center at $O$.

If $\Phi$ is a jacobian polynomial map, Vorob'ev theorem gives a class of polynomial hamiltonian isochronous centers. Next theorem was proved by Sabatini [88].

TheOREM 7.5. Let $\Phi \in C^{1}\left(\mathbf{R}^{2}, \mathbf{R}^{2}\right)$ be a polynomial jacobian map such that $\Phi(0,0)=(0,0)$. Then the origin is a global center of system (44) if and only if $\Phi$ is a global diffeomorphism of the plane onto itself.

By theorem 7.5, it is equivalent to prove the Jacobian Conjecture in $\mathbf{R}^{2}$ and to prove that a critical point of every system of the type (44), under the assumption that $J_{\Phi} \equiv c \neq 0$, is a global isochronous center.

For a study of the relationship between isochronous singular points and the jacobian conjecture in a complex setting, see Gavrilov [49].

If $\Phi$ is a jacobian map, then the corresponding hamiltonian system (44) has odd degree. In Mardešić, Moser-Jauslin and Rousseau [67] it is proved the hamiltonian systems obtained from a quadratic jacobian map are the only cubic hamiltonian systems with a Darboux linearization. In fact, for every system arising from a jacobian map $\Phi, \Phi$ is a linearization (see Sabatini [88]). In case $\Phi$ is injective, it is a global linearization.

Cubic hamiltonian systems with isochronous centers have been classified by Cima, Mañosas and Villadelprat [32]. Every cubic hamiltonian system with an isochronous center at the origin is of the type (44), with $\Phi$ jacobian quadratic map (see Meisters [71], for a classification of such polynomial maps). Cima, Mañosas and Villadelprat [32] give also an example of a 
TABLE 2.

Hamiltonian Systems.

System H milton
Hypothesis:
$\frac{\partial P}{\partial x} \frac{\partial Q}{\partial y}-\frac{\partial P}{\partial y} \frac{\partial Q}{\partial x} \equiv$ const. $\neq 0$.
Cartesian coordinates:
$\dot{x}=P \frac{\partial P}{\partial y}+Q \frac{\partial Q}{\partial y}$
$\dot{y}=-P \frac{\partial P}{\partial x}-Q \frac{\partial Q}{\partial x}$.
Transversal commuting system:
$\dot{x}=P \frac{\partial Q}{\partial y}-Q \frac{\partial P}{\partial y}$
$\dot{y}=-P \frac{\partial Q}{\partial x}+Q \frac{\partial P}{\partial x}$.
Linearization:
$\chi=P(x, y)$,
$\eta=Q(x, y)$.
Strong first integral:
$I=P^{2}+Q^{2}$,
$V=P P^{2}+Q{ }^{2}$.

hamiltonian system with an isochronous, polynomial, non-global center. The corresponding hamiltonian function is

$$
H(x, y)=\frac{x^{2}}{2}+x^{2}\left(x^{2}+1\right) y+\frac{1}{2}\left(x^{2}+1\right)^{3} y^{2} .
$$

In [32] the authors also give a linearization of the above system, that is defined on all of $\mathbf{R}^{2}$, even if the center is not a global one. The linearization is not rational.

In next table we collect some facts about isochronous centers of the type considered in theorem 7.5. It could appear weird that we give a nonconstant integrating factor for a system with a known first integral $H(x, y)$. Indeed, the function $\frac{1}{P^{2}+Q^{2}}$ is an integrating factor both for the hamiltonian system and for its commutator. The first integral of the commutator one gets in this way is a multivalued function.

A result of non-existence of isochronous centers for a special class of hamiltonians was proved by Christopher and Devlin [28] and Schumann 
[94]. They proved that a hamiltonian system generated by a polynomial hamiltonian function of the type

$$
H(x, y)=Q(x, y)+N(x, y)
$$

with $Q(x, y)$ quadratic, and $N(x, y)$ homogeneous of degree $>2$, cannot have an isochronous center at the origin. For the same systems, Gasull, Guillamon, Mañosa and Mañosas [46] prove that also singular points different from the origin cannot be isochronous. For a weaker result in the same direction, see Collins [33].

Another result in the same direction was obtained by Villadelprat [103], by proving that every analytic hamiltonian $H$ that generates an isochronous center can be written as the sum of two squares

$$
H(x, y)=\frac{1}{2}\left(f(x, y)^{2}+g(x, y)^{2}\right)
$$

where $f$ and $g$ are the components of an analytic area-preserving trasformation.

\section{UNIFORMLY ISOCHRONOUS CENTERS}

If all the solutions of system (1) rotate around the origin, and their angular speed is constant along rays, finding isochronous centers is equivalent to proving the existence of centers. This fact motivates the following definition.

DEFINITION 8.1. We say that the origin is a uniformly isochronous center of system (1) if it is a center and, in polar coordinates $x=\rho \cos (\varphi)$, $y=\rho \sin (\varphi),(1)$ takes the form

$$
\dot{\rho}=\alpha(\rho, \varphi), \quad \dot{\varphi}=\beta(\varphi) \text {. }
$$

Several systems in this class will be considered in next sections: $S_{2}, S_{2}^{*}$, $C R_{2}, H 4_{1}, H 5_{1}$. In this section we collect some results that hold for systems of arbitrary degree.

Polynomial systems with $\dot{\varphi}$ independent of $r$ have been studied by Conti in [36], showing that the only polynomial systems having uniformly isochronous centers are those ones with $\dot{\varphi}=k \in \mathbf{R}, k \neq 0$. Polynomial systems with an uniformly isochronous center can always be written in the following form

$$
\dot{x}=y+x P(x, y), \quad \dot{y}=-x+y P(x, y),
$$

where $P$ is a polynomial. 
System (46) can neither be a hamiltonian, nor a complex system. Let us set:

$$
P(x, y)=\sum_{l=0}^{n-1} p_{l} x^{n-1-l} y^{l},
$$

where $n$ is the degree of (46). The following theorem is due to Conti [36] and characterizes systems (46) with a center, when $P(x, y)$ is a homogeneous polynomial (see also [28], [70]).

Theorem 8.1. Let $P(x, y)$ be a homogeneous polynomial. Then the origin is an isochronous center of system (46) if and only if one of the following is true:

(i) system (46) has even degree;

(ii) system (46) has odd degree $n=2 m+1$, and

$$
\sum_{l=0}^{2 m} p_{l} \int_{0}^{2 \pi}(\cos \varphi)^{2 m-l} \varphi(\sin \varphi)^{l} d \varphi=0
$$

When $O$ is an isochronous center of system (46), the orbits of (46) are contained in algebraic curves. Moreover, $O$ cannot be a global center of (46) [36].

Collins [34] has studied a class of systems of the type (46), with $P(x, y)=$ $\alpha x+\beta y+A x^{2}+B x y+C y^{2}$. He has proved that such systems have an isochronous center at $O$ if and only if

$$
A+C=0, \quad A \alpha^{2}+B \alpha \beta+C \beta^{2}=0 .
$$

In this case there exists a rotation taking the system into a system of the form

$$
\dot{x}=-y+x^{2}(a+b y), \quad \dot{y}=x+x y(a+b y),
$$

where $a, b \in \mathbf{R}$. Linearizations of such systems have been given in Mardešić, Rousseau and Toni [68].

Uniformly isochronous systems - not necessarily polynomial ones - have been studied also in Mazzi and Sabatini [70], looking for commutators and linearizations of systems of the type

$$
\dot{x}=-y+x H(x, y), \quad \dot{y}=x+y H(x, y),
$$

where $H(0,0)=0$. Since system (49), can be seen as a linear system perturbed by a radial nonlinearity, one can look for a commutator of the 
same type:

$$
\dot{x}=x+x K(x, y), \quad \dot{y}=y+y K(x, y) .
$$

In this case the commutativity condition reduces to a single partial differential equation in $H$ and $K$ :

$x\left(\frac{\partial K}{\partial y}-\frac{\partial H}{\partial x}+H \frac{\partial K}{\partial x}-K \frac{\partial H}{\partial x}\right)+y\left(-\frac{\partial K}{\partial x}-\frac{\partial H}{\partial y}+H \frac{\partial K}{\partial y}-K \frac{\partial H}{\partial y}\right)=0$

so that computations are greatly simplified.

Even if one does not know all the solutions of equation (51), for every couple of $H$ and $K$ satisfying equation (51) one can give a linearization of both system (49) and system (50), and a first integral of system (49). Theorems 8.2-8.5 have been proved in [70]. Let us set

$$
\Omega(x, y)=\Omega(r \cos \varphi, r \sin \varphi)=\exp \left(-\int_{0}^{r} \frac{K(s \cos \varphi, s \sin \varphi)}{s(1+K(s \cos \varphi, s \sin \varphi))} d s\right) .
$$

THEOREM 8.2. Let $H(x, y)$ and $K(x, y)$ satisfy the equation (51). Then the transformation

$$
\chi(x, y)=x \Omega(x, y), \quad \eta(x, y)=y \Omega(x, y),
$$

linearizes both (49) and (50). Moreover, the function

$$
I(x, y)=\left(x^{2}+y^{2}\right) \Omega(x, y)^{2}
$$

is a first integral of (49).

In next theorem a couple of solutions to the partial differential equation (51) is provided.

TheOrem 8.3. Let $H(x, y)=x \sigma(y)$ and $K(x, y)=y \sigma(y)$, where $\sigma(y)$ is a function of class $C^{2}$. Then system (49) and system (50) commute, and $O$ is an isochronous center of system (49).

For $\sigma(y)=a$, one obtains one of Loud quadratic centers (see system $S_{2}$, section 10), while for $\sigma(y)=(a+b y)$ one obtains Collins' systems (see system $C R_{2}$, section 12). The linearization and the first integral for this special case are given in next table. 
TABLE 3.

System $\boldsymbol{U}_{\boldsymbol{\sigma}}$.

System $U_{\sigma}$

Cartesian coordinates:

$\dot{x}=-y+x^{2} \sigma(y)$,

$\dot{y}=x+x y \sigma(y)$.

Polar coordinates:

$\dot{r}=r^{2} \cos \varphi \sigma(r \sin \varphi)$, $\dot{\varphi}=1$.

Transversal commuting system

$\dot{x}=x+x y \sigma(y)$,

$\dot{y}=y+y^{2} \sigma(y)$.

$$
\begin{gathered}
\text { Linearization } \\
\chi=x \exp \left(-\int_{0}^{y} \frac{\sigma(s)}{1+s \sigma(s)} d s\right), \\
\eta=y \exp \left(-\int_{0}^{y} \frac{\sigma(s)}{1+s \sigma(s)} d s\right) .
\end{gathered}
$$

Strong first integral:

$H=\left(x^{2}+y^{2}\right) \exp \left(-\int_{0}^{y} \frac{2 \sigma(s)}{1+s \sigma(s)} d s\right)$.

Reciprocal integrating factor:

$V=\left(x^{2}+y^{2}\right)(1+y \sigma(y))$. 
The structure of equation (51) allows to give other classes of special solutions. In particular, if $H$ and $K$ are conjugate harmonic functions, the first two terms in each of the parentheses in equation (51) vanish. In this case we have a necessary and sufficient condition for system (50) to be a commutator of system (49).

THEOREM 8.4. Let $H$ and $K$ be conjugate harmonic functions. Then system (49) and system (50) commute if and only if there exists a function $\delta$, such that

$$
H^{2}(x, y)+K^{2}(x, y)=\delta\left(x^{2}+y^{2}\right)
$$

Another possibility arises assuming $H$ and $K$ to be homogeneous functions of the same degree $d \geq 1$. In this case the commutativity condition is very simple

$$
x \frac{\partial K}{\partial y}-y \frac{\partial K}{\partial x}=d H
$$

If $H$ is a homogeneous harmonic function, then the origin is an isochronous center (see Mazzi and Sabatini [70]).

THEOREM 8.5. Let $H$ and $K$ be homogeneous conjugate harmonic functions. Then system (49) and system (50) commute and $O$ is an isochronous center.

This means that if $H$ is a homogeneous and harmonic polynomial, Conti's conditions are satisfied (see theorem 8.1). The same conditions for systems with homogeneous nonlinearities have been found in Mazzi and Sabatini [70] by the commutator approach. In this case the linearization assumes the simple form

$$
\chi=x(1+K(x, y))^{-1 / d}, \quad \eta=y(1+K(x, y))^{-1 / d},
$$

where $d$ is the homogeneity degree of $H$. A first integral is

$$
I(x, y)=\frac{\left(x^{2}+y^{2}\right)^{d}}{(1+K(x, y))^{2}} .
$$

\section{NON-HAMILTONIAN SECOND ORDER O.D.E.'S}

In this section we are concerned with second order differential equations which are not equivalent to systems of the type (39). Volokitin and 
Ivanov [106] have proved that, for every positive integer $m$, the origin is an isochronous center of the equation

$$
\ddot{x}+x^{2 m-1} \dot{x}\left(x^{2}+\dot{x}^{2}\right)+x=0 .
$$

They also proved that every polynomial commutator of the system

$$
\dot{x}=y, \quad \dot{y}=-x-x^{2 m-1} y\left(x^{2}+y^{2}\right),
$$

equivalent to (59), can be obtained from (60) by multiplying by a suitable real constant. That is, (60) has only trivial polynomial commutators.

Volokitin and Ivanov [106] prove also a similar result for the so-called Newtonian systems

$$
\dot{x}=y, \quad \dot{y}=q_{0}(x)+q_{1}(x) y+\ldots+q_{n}(x) y^{n},
$$

where $n$ is a positive integer, and $q_{0}(0)=0$.

THEOREM 9.1. If $n=2$ or $n \geq 4$, then (61) does not have non-trivial polynomial commutators.

The case $n=1$ corresponds to the so-called Liénard differential equation

$$
\ddot{x}+f(x) \dot{x}+g(x)=0,
$$

that appears in several problems in applied mathematics. It has been the object of several investigations, and has often provided motivations for the development of new techniques (see $[10,92,72]$ ). We assume $g(0)=0$, so that the origin is a critical point for the equivalent plane system

$$
\dot{x}=y, \quad \dot{y}=-g(x)-y f(x) .
$$

Cherkas [24] gave necessary and sufficient conditions for the origin to be a center of (63), when $f$ and $g$ are analytic functions (see also Gasull and Torregrosa [48] for an extension). On the other hand, only recently the isochronicity problem received significant contributions. Next theorem has been independently proved by Algaba, Freire and Gamero [2] in the polynomial case, by Christopher, Devlin and Lloyd [29] and Sabatini [90] in the analytic case. We recall that if $f(x)$ and $g(x)$ are odd, then (63) is reversible with respect to the $y$ axis.

THEOREM 9.2. Let $f(x)$ and $g(x)$ be odd analytic functions, with $x g(x)>$ 0 in a neighbourhood of the origin. Then $O$ is an isochronous center of (63) if and only if $g^{\prime}(0)>0$ and:

$$
g(x)=g^{\prime}(0) x+\frac{1}{x^{3}}\left(\int_{0}^{x} s f(s) d s\right)^{2} .
$$


If $f(x)$ and $g(x)$ are odd functions of class $C^{1}$, then the equality (64) is a sufficient condition for the isochronicity of $O$ [90]. In fact, in this case one can study the solutions of (62) by means of the non-traditional equivalent plane system

$$
\dot{x}=y-x B(x), \quad \dot{y}=-x-y B(x) .
$$

where $x^{2} B(x)=\int_{0}^{x} s f(s) d s, C(x)=g(x)-x B^{2}(x)$. When $f(x)$ and $g(x)$ are odd, and (64) holds, the origin is a uniformly isochronous center of (65). If, additionally, $f(x)=-(k+2) x^{k}$ for some positive integer, one can find a polynomial commutator, a linearization and a rational first integral of (65) (see Mazzi and Sabatini [70]).

In the non-reversible case, the equality (64) cannot be a condition for the isochronicity of (63), since there exist analytic systems of type (63), with $f \equiv 0, g$ non-odd, and an isochronous center at the origin [101, 32].

In [2] several special cases of non-odd polynomial Liénard systems have been considered, proving, in those cases, the non-existence of isochronous centers when $f(x)$ and $g(x)$ do not satisfy the equality (64). In [29] the authors claim to have proved, by means of a computer assisted procedure, the non-existence of isochronous centers of the polynomial Liénard equation, if the degrees of $f(x)$ and $g(x)$ do not exceed 27. In both papers it is conjectured that isochronous centers of polynomial systems occur only when $f(x)$ and $g(x)$ are odd.

The case $n=3$ of newtonian systems corresponds to the so-called Abel systems. The following theorem is due to Volokitin and Ivanov [106].

TheOREM 9.3. A polynomial Abel system has a nontrivial polynomial commutator if and only if it has the form

$$
\dot{x}=y, \quad \dot{y}=-\left(x+a^{2} x^{3}\right)(1+h(x) y)^{3}-3 a x y(1+h(x) y)^{2}+h^{\prime}(x) y^{3},
$$

where $a \in \mathbf{R}$ and $h(x)$ is an arbitrary polynomial.

Hence, if (66) has a center, it has an isochronous center. The integrability of (66) is proved in the following theorem, that holds also for non-polynomial systems [106].

THEOREM 9.4. The change of variables

$$
X=x, \quad Y=\frac{y}{1+h(x) y},
$$

transforms (66) into the reparametrized Kukles system

$$
\dot{X}=\frac{Y}{1-h(X) Y}, \quad \dot{Y}=\frac{-X-3 a X Y-a^{2} X^{3}}{1-h(X) Y},
$$


that has a center at the origin.

For a very special case of a fourth-degree system with an isochronous center, equivalent to a second order differential equation, see Kononova $[57]$.

\section{QUADRATIC SYSTEMS}

Let us consider a quadratic system of the form

$$
\dot{x}=-y+a_{20} x^{2}+a_{11} x y+a_{02} y^{2}, \quad \dot{y}=x+b_{20} x^{2}+b_{11} x y+b_{02} y^{2} .
$$

In polar coordinates $x=r \cos (\varphi), y=r \sin (\varphi)$, we can write system (69) in the form

$$
\begin{aligned}
& \dot{r}=r^{2}\left(R_{3} \cos \left(3 \varphi+\varphi_{3}\right)+R_{1} \cos \left(\varphi+\varphi_{1}\right)\right), \\
& \dot{\varphi}=1+r\left(-R_{3} \sin \left(3 \varphi+\varphi_{3}\right)+r_{1} \sin \left(\varphi+\bar{\varphi}_{1}\right)\right) .
\end{aligned}
$$

Let the origin be a center. The problem of isochronicity of such system was studied by Loud [64]. He proved

THEOREM 10.1. The origin is an isochronous center of the quadratic system (69) if and only if the system can be brought to one of the following systems $\left(S_{1}\right),\left(S_{2}\right),\left(S_{3}\right),\left(S_{4}\right)$, through a linear change of coordinates and a rescaling of time.

One can find strong first integrals, linearizing change of coordinates and phase portraits in the Poincaré disc for these systems in Mardešić, Rousseau and Toni [68]. Sabatini [85] gave the transversal commuting systems for these systems.

\section{CUBIC SYSTEMS WITH HOMOGENEOUS NONLINEARITIES}

Let us consider a cubic system (3) of the form

$$
\begin{aligned}
& \dot{x}=-y+a_{30} x^{3}+a_{21} x^{2} y+a_{12} x y^{2}+a_{03} y^{3}, \\
& \dot{y}=x+b_{30} x^{3}+b_{21} x^{2} y+b_{12} x y^{2}+b_{03} y^{3} .
\end{aligned}
$$

In polar coordinates $x=r \cos (\varphi), y=r \sin (\varphi)$, we can write system (71) in the form

$$
\begin{aligned}
& \dot{r}=r^{3}\left(R_{4} \cos \left(4 \varphi+\varphi_{4}\right)+R_{2} \cos \left(\varphi+\varphi_{2}\right)+R_{0}\right), \\
& \dot{\varphi}=1+r^{2}\left(-R_{4} \sin \left(4 \varphi+\varphi_{4}\right)+r_{2} \sin \left(\varphi+\bar{\varphi}_{2}\right)+r_{0}\right) .
\end{aligned}
$$


TABLE 4.

Quadratic Systems.

\begin{tabular}{|c|c|}
\hline System $\mathbf{S}_{1}$ & System $\mathbf{S}_{2}$ \\
\hline $\begin{array}{c}\text { Cartesian coordinates: } \\
\dot{x}=-y+x^{2}-y^{2}, \dot{y}=x(1+2 y) .\end{array}$ & $\begin{array}{c}\text { Cartesian coordinates: } \\
\dot{x}=-y+x^{2}, \dot{y}=x(1+y) .\end{array}$ \\
\hline $\begin{array}{c}\text { Polar coordinates: } \\
\dot{r}=r^{2} \cos \varphi, \dot{\varphi}=1+r \sin \varphi .\end{array}$ & $\begin{array}{l}\text { Polar coordinates: } \\
\dot{r}=r^{2} \cos \varphi, \dot{\varphi}=1\end{array}$ \\
\hline $\begin{array}{l}\text { Strong first integral: } \\
\qquad H=\frac{x^{2}+y^{2}}{1+2 y}\end{array}$ & $\begin{array}{l}\text { Strong first integral: } \\
\qquad H=\frac{x^{2}+y^{2}}{(1+y)^{2}}\end{array}$ \\
\hline $\begin{array}{l}\text { Reciprocal integrating factor: } \\
\qquad V=(1+2 y)^{2}\end{array}$ & $\begin{array}{l}\text { Reciprocal integrating factor: } \\
\qquad V=(1+y)^{3}\end{array}$ \\
\hline $\begin{array}{c}\text { Linearization: } \\
\zeta=\frac{z}{z+i}, \text { where } z=x+i y\end{array}$ & $\begin{array}{l}\text { Linearization: } \\
\chi=\frac{x}{1+y}, \eta=\frac{y}{1+y}\end{array}$ \\
\hline $\begin{array}{l}\text { Transversal commuting system: } \\
\dot{x}=x(1+2 y), \dot{y}=y-x^{2}+y^{2} .\end{array}$ & $\begin{array}{l}\text { Transversal commuting system: } \\
\quad \dot{x}=x(1+y), \dot{y}=y(1+y)\end{array}$ \\
\hline System $\mathbf{S}_{3}$ & System $\mathbf{S}_{4}$ \\
\hline $\begin{array}{c}\text { Cartesian coordinates: } \\
\dot{x}=-y-\frac{4}{3} x^{2}, \dot{y}=x\left(1-\frac{16}{3} y\right) .\end{array}$ & $\begin{array}{c}\text { Cartesian coordinates: } \\
\dot{x}=-y+\frac{16}{3} x^{2}-\frac{4}{3} y^{2}, \dot{y}=x\left(1+\frac{8}{3} y\right) .\end{array}$ \\
\hline $\begin{array}{c}\text { Polar coordinates: } \\
\dot{r}=r^{2}\left(\cos 3 \varphi-\frac{7}{3} \cos \varphi\right), \\
\dot{\varphi}=1+r(-\sin 3 \varphi-\sin \varphi)\end{array}$ & $\begin{array}{c}\text { Polar coordinates: } \\
\dot{r}=r^{2}\left(\cos 3 \varphi+\frac{13}{3} \cos \varphi\right) \\
\dot{\varphi}=1+r\left(-\sin 3 \varphi+\frac{1}{3} \sin \varphi\right)\end{array}$ \\
\hline $\begin{array}{c}\text { Strong first integral: } \\
H=\frac{9\left(x^{2}+y^{2}\right)-24 x^{2} y+16 x^{4}}{-3+16 y} .\end{array}$ & $\begin{array}{l}\text { Strong first integral: } \\
H=\frac{9\left(x^{2}+y^{2}\right)+24 y^{3}+16 y^{4}}{(3+8 y)^{4}} .\end{array}$ \\
\hline $\begin{array}{l}\text { Reciprocal integrating factor: } \\
V=(3-16 y)\left(9-24 y+32 x^{2}\right) .\end{array}$ & $\begin{array}{c}\text { Reciprocal integrating factor: } \\
V=(3+8 y)\left(9+96 y-256 x^{2}+128 y^{2}\right) .\end{array}$ \\
\hline $\begin{array}{l}\text { Linearization: } \\
\chi=\frac{3 x}{9-24 y+32 x^{2}}, \eta=\frac{3 y-4 x^{2}}{9-24 y+32 x^{2}} .\end{array}$ & $\begin{array}{c}\text { Linearization: } \\
\chi=\frac{3 x}{(3+8 y)^{2}}, \eta=\frac{3 y+4 y^{2}}{(3+8 y)^{2}} .\end{array}$ \\
\hline $\begin{array}{l}\text { Transversal commuting system: } \\
\quad \dot{x}=3 x\left(9-24 y+32 x^{2}\right) \\
\dot{y}=\left(3 y+4 x^{2}\right)\left(9-24 y+32 x^{2}\right) .\end{array}$ & $\begin{array}{l}\text { Transversal commuting system: } \\
\quad \dot{x}=x(3+8 y)^{2} \\
\dot{y}=y(3+4 y)(3+8 y)\end{array}$ \\
\hline
\end{tabular}


TABLE 5.

Homogeneous Cubic Systems (I).

\begin{tabular}{|c|c|}
\hline System $\mathbf{S}_{1}^{*}$ & System $\mathrm{S}_{2}^{*}$ \\
\hline $\begin{array}{c}\text { Cartesian coordinates: } \\
\dot{x}=-y+x^{3}-3 x y^{2}, \dot{y}=x+3 x^{2} y-y^{3}\end{array}$ & $\begin{array}{c}\text { Cartesian coordinates: } \\
\dot{x}=-y+x^{3}-x y^{2}, \dot{y}=x+x^{2} y-y^{3} .\end{array}$ \\
\hline $\begin{array}{c}\text { Polar coordinates: } \\
\dot{r}=r^{3} \cos 2 \varphi, \dot{\varphi}=1+r^{2} \sin 2 \varphi .\end{array}$ & $\begin{array}{l}\text { Polar coordinates: } \\
\dot{r}=r^{3} \cos 2 \varphi, \dot{\varphi}=1\end{array}$ \\
\hline $\begin{array}{l}\text { Strong first integral: } \\
\qquad H=\frac{\left(x^{2}+y^{2}\right)^{2}}{1+4 x y}\end{array}$ & $\begin{array}{l}\text { Strong first integral: } \\
\qquad H=\frac{x^{2}+y^{2}}{1+2 x y}\end{array}$ \\
\hline $\begin{array}{l}\text { Reciprocal integrating factor: } \\
\qquad V=\left(x^{2}+y^{2}\right)^{3}\end{array}$ & $\begin{array}{l}\text { Reciprocal integrating factor: } \\
\qquad V=(1+2 x y)^{2}\end{array}$ \\
\hline$\zeta=\frac{z}{\sqrt{i+z^{2}}}$, where $z=x+i y$ & $\begin{array}{c}\text { Linearization: } \\
\chi=\frac{x}{\sqrt{1+2 x y}}, \eta=\frac{y}{\sqrt{1+2 x y}}\end{array}$ \\
\hline $\begin{array}{c}\text { Transversal commuting system: } \\
\dot{x}=x+3 x^{2} y-y^{3}, \dot{y}=y-x^{3}+3 x y^{2} .\end{array}$ & $\begin{array}{l}\text { Transversal commuting system: } \\
\dot{x}=x(1+2 x y), \dot{y}=y(1+2 x y) \text {. }\end{array}$ \\
\hline
\end{tabular}

Let the origin be a center. The problem of isochronicity of such system was studied by Pleshkan [76]. He proved the following theorem.

THEOREM 11.1. The origin is an isochronous center of cubic system (71) if and only if the system can be brought to one of the following systems $\left(S_{1}^{*}\right)$, $\left(S_{2}^{*}\right),\left(S_{3}^{*}\right),\left(\tilde{S}_{3}^{*}\right)$, through a linear change of coordinates and a rescaling of time.

One can find strong first integrals, linearizing change of coordinates and phase portraits in the Poincaré disc for these systems in Mardešić, Rousseau and Toni [68]. Mazzi and Sabatini [70] and Gasull, Guillamón and Mañosa [45] gave transversal commuting systems for these systems. 
TABLE 6.

Homogeneous Cubic Systems (II).

\begin{tabular}{|c|c|}
\hline System $\mathbf{S}_{3}^{*}$ & System $\mathbf{S}_{3}^{*}$ \\
\hline $\begin{array}{c}\text { Cartesian coordinates: } \\
\dot{x}=-y+3 x^{2} y, \dot{y}=x-2 x^{3}+9 x y^{2}\end{array}$ & $\begin{array}{c}\text { Cartesian coordinates: } \\
\dot{x}=-y-3 x^{2} y, \dot{y}=x+2 x^{3}-9 x y^{2} .\end{array}$ \\
\hline $\begin{array}{c}\text { Polar coordinates: } \\
\dot{r}=r^{3}\left(-\sin 4 \varphi+\frac{5}{2} \sin 2 \varphi\right), \\
\dot{\varphi}=1-r^{2}(\cos 4 \varphi+\cos 2 \varphi) .\end{array}$ & $\begin{array}{c}\text { Polar coordinates: } \\
\dot{r}=r^{3}\left(\sin 4 \varphi-\frac{5}{2} \sin 2 \varphi\right), \\
\dot{\varphi}=1+r^{2}(\cos 4 \varphi+\cos 2 \varphi) .\end{array}$ \\
\hline $\begin{array}{l}\text { Strong first integral: } \\
H=\frac{x^{2}+y^{2}-4 x^{4}+4 x^{6}}{\left(-1+3 x^{2}\right)^{3}} .\end{array}$ & $\begin{array}{l}\text { Strong first integral: } \\
H=\frac{x^{2}+y^{2}+4 x^{4}+4 x^{6}}{\left(1+3 x^{2}\right)^{3}} .\end{array}$ \\
\hline $\begin{array}{l}\text { Reciprocal integrating factor: } \\
\qquad V=\left(1-3 x^{2}\right)^{4}\end{array}$ & $\begin{array}{l}\text { Reciprocal integrating factor: } \\
\qquad V=\left(1+3 x^{2}\right)^{4}\end{array}$ \\
\hline $\begin{array}{l}\quad \text { Linearization: } \\
\chi=\frac{x-2 x^{3}}{\left(1-3 x^{2}\right)^{\frac{3}{2}}}, \eta=\frac{y}{\left(1-3 x^{2}\right)^{\frac{3}{2}}}\end{array}$ & $\begin{array}{l}\text { Linearization: } \\
\chi=\frac{x+2 x^{3}}{\left(1+3 x^{2}\right)^{\frac{3}{2}}}, \eta=\frac{y}{\left(1+3 x^{2}\right)^{\frac{3}{2}}}\end{array}$ \\
\hline $\begin{array}{l}\text { Transversal commuting system: } \\
\begin{array}{c}\dot{x}=x\left(1-3 x^{2}\right)\left(1-2 x^{2}\right) \\
\dot{y}=y\left(1-3 x^{2}\right)\left(1-6 x^{2}\right)\end{array}\end{array}$ & $\begin{array}{l}\text { Transversal commuting system: } \\
\qquad \begin{array}{c}\dot{x}=x\left(1+3 x^{2}\right)\left(1+2 x^{2}\right) \\
\dot{y}=y\left(1+3 x^{2}\right)\left(1+6 x^{2}\right)\end{array}\end{array}$ \\
\hline
\end{tabular}




\section{CUBIC REVERSIBLE SYSTEMS}

In this section we study isochronous centers of cubic systems reversible with respect to a line passing through the origin. The general form of this systems in polar coordinates is given in Theorem 4.1 (ii). In the particular case $m=3$, we have

$$
\begin{aligned}
& \dot{r}=r^{2}\left(R_{3} \sin 3 \omega+R_{1} \sin \omega\right)+r_{3}\left(R_{4} \sin 4 \omega+R_{2} \sin 2 \omega\right), \\
& \dot{\omega}=1+r\left(R_{3} \cos 3 \omega+r_{1} \cos \omega\right)+r^{2}\left(R_{4} \cos 4 \omega+r_{2} \cos 2 \omega+r_{0}\right),
\end{aligned}
$$

where $R_{4}, R_{3}, R_{2}, R_{1}, r_{2}, r_{1}$, and $r_{0}$ are arbitrary coefficients.

A general theory for such systems has not yet been developped. There are partial results concerning particular cases. The results of this section are due to Chavarriga and García [12].

12.1. Cubic Reversible Systems with $\boldsymbol{R}_{3}=0$ and $\boldsymbol{R}_{4} \neq 0$.

Let us consider system (73) with $R_{3}=0$ and $R_{4} \neq 0$. The results of this section were obtained by means of complex computer algebra procedures. The outcome of such computations can be summarized in next statement. Statement 1. The only reversible cubic isochronous systems (73) with $R_{3}=0$ and $R_{4} \neq 0$ are the cubic isochronous systems given by Theorem 11.1.

12.2. Cubic Reversible Systems with $\boldsymbol{R}_{3}=\boldsymbol{R}_{4}=0$.

Let us consider system (73) with $R_{3}=R_{4}=0$. The following result holds.

THEOREM 12.1. The origin is an isochronous center of cubic system (73), reversible with respect to a line passing through the origin, with $R_{3}=0$ and $R_{4}=0$ if and only if system (73) can be brought to one of the following systems $\left(C R_{1}\right),\left(C R_{2}\right),\left(C R_{3}\right),\left(C R_{4}\right)$ and $\left(C R_{5}\right)$ by a linear change of coordinates and a rescaling of time.

12.3. Cubic Reversible Hamiltonian Systems.

If system (73) is hamiltonian, that is if $r_{1}=3 R_{1}$ and $r_{2}=2 R_{2}$, the following result holds. 
TABLE 7.

Cubic Reversible Systems $\mathbf{C R}_{\mathbf{1}}$ and $\mathbf{C R}_{\mathbf{2}}$.

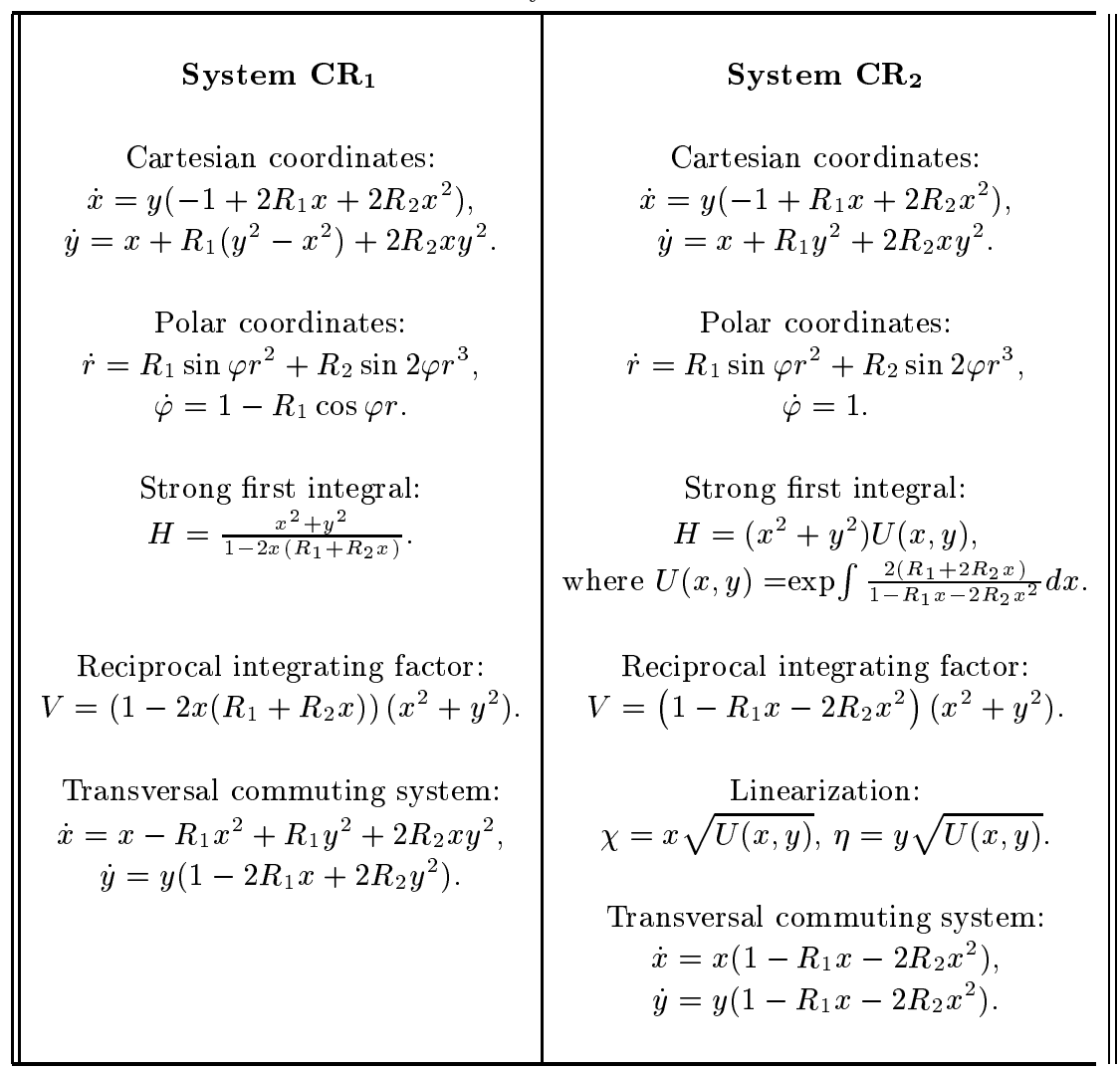


TABLE 8.

Cubic Reversible System $\mathbf{C R}_{\mathbf{3}}$.

\section{System $\mathbf{C R}_{3}$}

Cartesian coordinates:

$\dot{x}=y\left(-1+2 R_{1} x+3 R_{2} x^{2}-R_{2} y^{2}\right), \dot{y}=x-R_{1} x^{2}+R_{1} y^{2}-R_{2} x^{3}+3 R_{2} x y^{2}$

Polar coordinates:

$\dot{r}=R_{1} \sin \varphi r^{2}+R_{2} \sin 2 \varphi r^{3}, \dot{\varphi}=1-R_{1} \cos \varphi r-R_{2} \cos 2 \varphi r^{2}$.

Strong first integral:

$H=\prod_{i=1}^{3} f_{i}^{\lambda_{i}}$, where $f_{1}=\left(R_{1}-\Delta\right)^{2}+R_{2}\left(R_{1}-\Delta\right) x+R_{2}^{2}\left(x^{2}+y^{2}\right)$, $f_{2}=\left(R_{1}+\Delta\right)^{2}+R_{2}\left(R_{1}+\Delta\right) x+R_{2}^{2}\left(x^{2}+y^{2}\right), f_{3}=x^{2}+y^{2}$ and

$\Delta:=\sqrt{R_{1}^{2}+4 R_{2}}, \lambda_{1}=-1-R_{1} / \Delta, \lambda_{2}=-1+R_{1} / \Delta, \lambda_{3}=2$.

Reciprocal integrating factor:

$V=\left(x^{2}+y^{2}\right)\left(-1+2 R_{1} x-R_{1}^{2}\left(x^{2}+y^{2}\right)+2 R_{2}\left(x^{2}-y^{2}\right)\right.$ $\left.-2 R_{1} R_{2} x\left(x^{2}+y^{2}\right)-R_{2}^{2}\left(x^{2}+y^{2}\right)^{2}\right)$.

Transversal commuting system:

$\dot{x}=x-R_{1} x^{2}+R_{1} y^{2}-R_{2} x^{3}+3 R_{2} x y^{2}, \dot{y}=y\left(1-2 R_{1} x-3 R_{2} x^{2}+R_{2} y 2\right)$. 
TABLE 9.

Cubic Reversible System $\mathbf{C R}_{4}$.

\section{System CR $_{4}$}

Cartesian coordinates:

$$
\begin{gathered}
\dot{x}=y\left(-1+\left(R_{1}-r_{1}\right) x+r_{1}\left(r_{1}+R_{1}\right)\left(x^{2}-y^{2}\right)\right), \\
\dot{y}=x+r_{1} x^{2}+R_{1} y^{2}+2 r_{1}\left(r_{1}+R_{1}\right) x y^{2} .
\end{gathered}
$$

Polar coordinates:

$$
\begin{gathered}
\dot{r}=R_{1} \sin \varphi r^{2}+\frac{1}{2} r_{1}\left(r_{1}+R_{1}\right) \sin 2 \varphi r^{3}, \\
\dot{\varphi}=1+r_{1} \cos \varphi r+\frac{1}{2} r_{1}\left(r_{1}+R_{1}\right)(1-\cos 2 \varphi) r^{2} .
\end{gathered}
$$

Strong first integral:

$$
\begin{gathered}
H=\left(x^{2}+y^{2}\right)\left(1+2 r_{1} x+r_{1}^{2}\left(x^{2}+y^{2}\right)\right)^{\frac{R_{1}-r_{1}}{3 r_{1}+R_{1}}}\left(r_{1}-R_{1}+\left(R_{1}^{2}-r_{1}^{2}\right) x+\right. \\
\left.+r_{1}\left(r_{1}+R_{1}\right)^{2}\left(x^{2}+y^{2}\right)\right)^{-\frac{2\left(r_{1}+R_{1}\right)}{3 r_{1}+R_{1}}}, \text { if } R_{1} \neq r_{1} ; \\
H=\frac{x^{2}+y^{2}}{1+2 r_{1} x+r_{1}^{2}\left(x^{2}+y^{2}\right)} \exp \left[\frac{2 r_{1} x-1}{r_{1}^{2}\left(x^{2}+y^{2}\right)}\right] \text { if } R_{1}=r_{1} \text {. Notice that } H \in C^{\infty} .
\end{gathered}
$$

Reciprocal integrating factor:

$V=\left(x^{2}+y^{2}\right)\left(1+2 r_{1} x+r_{1}^{2}\left(x^{2}+y^{2}\right)\right)\left(r_{1}-R_{1}+\left(R_{1}^{2}-r_{1}^{2}\right) x+r_{1}\left(r_{1}+R_{1}\right)^{2}\left(x^{2}+y^{2}\right)\right)$.

Transversal commuting system:

$$
\begin{aligned}
& \int \dot{x}=x-R_{1} x^{2}-r_{1} y^{2}+2 r_{1} \frac{r_{1}+R_{1}}{r_{1}-R_{1}} x\left(R_{1} x^{2}+r_{1} y^{2}\right)+r_{1}^{2} \frac{\left(r_{1}+R_{1}\right)^{2}}{r_{1}-R_{1}}\left(x^{4}-y^{4}\right), \\
& \dot{y}=y\left(1+\left(r_{1}-R_{1}\right) x+r_{1} \frac{r_{1}+R_{1}}{r_{1}-R_{1}}\left(\left(3 R_{1}-r_{1}\right) x^{2}+\right.\right. \\
& \left.\left.+\left(r_{1}+R_{1}\right) y^{2}\right)+\frac{2 r_{1}^{2}\left(r_{1}+R_{1}\right)^{2}}{r_{1}-R_{1}} x\left(x^{2}+y^{2}\right)\right) \\
& \dot{x}=\left(x^{2}+y^{2}\right)\left(x+r_{1}\left(x^{2}-y^{2}\right)\right), \dot{y}=y\left(x^{2}+y^{2}\right)\left(1+2 r_{1} x\right) \text { if } R_{1}=r_{1} .
\end{aligned}
$$


TABLE 10.

Cubic Reversible System CR5.

System CR5
Cartesian coordinates:
$\dot{x}=-y(1-x)(1-2 x), \dot{y}=x-2 x^{2}+y^{2}+2 x^{3}$.
Polar coordinates:
$\dot{r}=\sin \varphi r^{2}$,
$\dot{\varphi}=1-2 \cos \varphi r+(1+\cos 2 \varphi) r^{2}$.
Strong first integral:
$H=\frac{(x-1)^{2}\left(x^{2}+y^{2}\right)}{(2 x-1)^{2}}$.
Reciprocal integrating factor:
$V=(1-x)(1-2 x)\left(x^{2}+y^{2}\right)$.
Transversal commuting system:
$\dot{x}=\frac{(1-x)\left(x-2 x^{2}+2 y^{2}+2 x^{3}-2 x y^{2}\right)}{1-2 x}$,
$\dot{y}=\frac{(1-x) y\left(1-4 x+6 x^{2}+2 y^{2}\right)}{1-2 x}$.


TABLE 11.

Hamiltonian cubic reversible system.

System CRH
Cartesian coordinates:
$\dot{x}=y\left(-1+\frac{8}{3} x-\frac{32}{9} y^{2}\right), \dot{y}=x-\frac{4}{3} y^{2}$.
Polar coordinates:
$\dot{r}=\left(-\frac{1}{3} \sin \varphi+\sin 3 \varphi\right) r^{2}+\frac{4}{9}(-2 \sin 2 \varphi+\sin 4 \varphi) r^{3}$,
$\dot{\varphi}=1+(-\cos \varphi+\cos 3 \varphi) r+\frac{1}{9}(12-16 \cos 2 \varphi+4 \cos 4 \varphi) r^{2}$.
Strong first integral, and reciprocal integrating factor:
$H=9 y^{2}+\left(3 x-4 y^{2}\right)^{2}$.
Linearization:
$\chi=x-\frac{4}{3} y^{2}, \eta=y$.
Transversal commuting system:
$\dot{x}=x+\frac{4}{3} y^{2}, \dot{y}=y$.

THEOREM 12.2. Every hamiltonian cubic system (73), reversible with respect to a line passing through the origin, having an isochronous center at $O$ can be transformed by a linear change of variables and a rescaling of time into system $(\mathrm{CRH})$.

\section{POLYNOMIAL SYSTEMS WITH HOMOGENEOUS NONLINEARITIES}

We consider the system

$$
\dot{x}=-y+X_{s}(x, y), \quad \dot{y}=x+Y_{s}(x, y),
$$

where $X_{s}(x, y)$ and $Y_{s}(x, y)$ are homogeneous polynomials of degree $s$, with $s \geq 2$.

By applying Lemma 4.1 we can write system (74) in polar coordinates, $x=r \cos \varphi, y=r \sin \varphi$, as

$$
\dot{r}=P_{s}(\varphi) r^{s}, \quad \dot{\varphi}=1+Q_{s}(\varphi) r^{s-1},
$$


where $P_{s}(\varphi)$ and $Q_{s}(\varphi)$ are trigonometric polynomials of the form (22).

In this section we give an account of the known isochronous centers of system (74) when $s=4$ and $s=5$.

\subsection{Computation of Isochronous Constants.}

Let the origin be an isochronous center. By theorem 3.3, there exists an analytic change of coordinates $\chi=x+o(|(x, y)|), \eta=y+o(|(x, y)|)$ such that $\ddot{\chi}+\chi=0$ and $\ddot{\eta}+\eta=0$, where we have taken $k=1$ making a rescaling of the time. Let $\chi=H(r, \varphi)$ a power series of the form

$$
H(r, \varphi)=H_{1}(\varphi) r+H_{2}(\varphi) r^{2}+H_{3}(\varphi) r^{3}+\ldots,
$$

where $H_{1}(\varphi)=\cos \varphi$ and $H_{i}(\varphi), i=1,2, \ldots$, are homogeneous trigonometric polynomials of degree $i$. Imposing $\ddot{H}+H=0$ we obtain a recursive system of differential equations given by

$$
\begin{aligned}
& H_{k}^{\prime \prime}+H_{k}=0, \quad k=1, \ldots, s-1, \\
& H_{(s-1)+k}^{\prime \prime}+H_{(s-1)+k}+2 Q_{s} H_{k}^{\prime \prime}+ \\
& \left(2 k P_{s}+Q_{s}^{\prime}\right) H_{k}^{\prime}+k P_{s}^{\prime} H_{k}=0 \text {, with } k=1, \ldots, s-1, \\
& H_{(m+2)(s-1)+k}^{\prime \prime}+H_{(m+2)(s-1)+k}+2 Q_{s} H_{(m+1)(s-1)+k}^{\prime \prime}+ \\
& \left(2((m+1)(s-1)+k) P_{s}+Q_{s}^{\prime}\right) H_{(m+1)(s-1)+k}^{\prime}+ \\
& ((m+1)(s-1)+k) P_{s}^{\prime} H_{(m+1)(s-1)+k}+Q_{s}^{2} H_{m(s-1)+k}^{\prime \prime}+ \\
& \left((2(m(s-1)+k)+(s-1)) P_{s} Q_{s}+Q_{s} Q_{s}^{\prime}\right) H_{m(s-1)+k}^{\prime}+ \\
& (m(s-1)+k)\left(((m+1)(s-1)+k) P_{s}^{2}+P_{s}^{\prime} Q_{s}\right) H_{m(s-1)+k}= \\
& \left\{\begin{array}{cc}
\alpha_{(m+2)(s-1)+k} \cos \varphi+\beta_{(m+2)(s-1)+k} \sin \varphi & \text { if }(m+2)(s-1)+k \text { is odd; } \\
0 & \text { if }(m+2)(s-1)+k \text { is even; }
\end{array}\right.
\end{aligned}
$$

where $k=1, \ldots, s-1, m=0,1, \ldots,{ }^{\prime}=\frac{d}{d \varphi}$ and $H_{1}(\varphi)=\cos \varphi$. The constants $\alpha_{(m+2)(s-1)+k}$ and $\beta_{(m+2)(s-1)+k}$ are the isochronous constants. These equations can be considered as $s-1$ families of independent differential equations.

Since the functions $H_{i}(\varphi)$ must be trigonometrical polynomials of degree $i$, the only possible solutions of equations (76) are

$$
H_{k}(\varphi)=\left\{\begin{array}{cl}
a_{k} \cos (\varphi)+b_{k} \sin (\varphi) & \text { if } k \text { is odd; } \\
0 & \text { if } k \text { is even; }
\end{array} k=1, \ldots, s-1,\right.
$$

where $a_{k}$ and $b_{k}, k=1, \ldots, s-1$, are real constants (in particular, $a_{1}=1$ and $\left.b_{1}=0\right)$. It is obvious that all equations of (76) are equal, and only 
the initial conditions change. Due to the special structure of the systems involved, we can assume, without any loss of generality, that $a_{1}=1, b_{1}=0$ and $a_{k}=b_{k}=0, k=3, \ldots, s-1$.

\subsection{Isochronous centers of a linear center perturbed by} homogeneous polynomials of degree 4

For $s=4$ system (75) takes the form

$$
\dot{r}=P_{4}(\varphi) r^{4}, \quad \dot{\varphi}=1+Q_{4}(\varphi) r^{3},
$$

where

$$
\begin{aligned}
& P_{4}(\varphi)=R_{5} \cos \left(5 \varphi+\varphi_{5}\right)+R_{3} \cos \left(3 \varphi+\varphi_{3}\right)+R_{1} \cos \left(\varphi+\varphi_{1}\right), \\
& Q_{4}(\varphi)=-R_{5} \sin \left(5 \varphi+\varphi_{5}\right)+r_{3} \sin \left(3 \varphi+\bar{\varphi}_{3}\right)+r_{1} \sin \left(\varphi+\bar{\varphi}_{1}\right) .
\end{aligned}
$$

The reversibility conditions for system (78) are $\bar{\varphi}_{1}=\varphi_{1}, \bar{\varphi}_{3}=\varphi_{3}, \varphi_{5}=$ $5 \varphi_{1}$ and $\varphi_{3}=3 \varphi_{1}$. The following theorem, due to Chavarriga, Giné and García [19], characterizes reversible isochronous centers of system (78).

THEOREM 13.1. A necessary condition for the origin of system (78) to be a reversible isochronous center is that the system can be brought to one of the following systems $\left(H 4_{1}\right),\left(H 4_{2}\right),\left(H 4_{3}\right),\left(H 4_{4}\right),\left(H 4_{5}\right),\left(H 4_{6}\right)$ and $\left(\mathrm{H}_{7}\right)$ through a linear change of coordinates and a rescaling of time. The condition is sufficient for systems $\left(H 4_{1}\right),\left(H 4_{2}\right),\left(H 4_{3}\right),\left(H 4_{4}\right),\left(H 4_{5}\right)$, $\left(\mathrm{H}_{6}\right)$.

For system $\left(\mathrm{H}_{7}\right)$, it is not known how to prove the isocronicity of the origin for all values of $r_{3}$. For this reason we are going to consider particular cases of this family.

First we consider some particular systems of family $\left(\mathrm{H}_{7}\right)$ that have a transversal commuting system. Chavarriga, Giné and García [19] have found three cases: for $r_{3}=\frac{9}{5}, r_{3}=-\frac{39}{5}$ and $r_{3}=-3$. These system have a reciprocal integrating factor of the form $V(x, y)=(P(x, y))^{b}$ where $P(x, y)$ is a polynomial in the variables $(x, y)$ such that $P(0,0) \neq 0$, and $b$ a rational number.

They have studied also the systems that have particular polynomial solutions and they have found that for $r_{3}=\frac{81}{5}$ and $r_{3}=-\frac{87}{5}$ there are algebraic 
TABLE 12.

Cubic reversible systems (I).

\section{System H41}

Cartesian coordinates:

$\dot{x}=-y+\left(R_{1}+R_{3}\right) x^{4}+\left(R_{1}-3 R_{3}\right) x^{2} y^{2}$, $\dot{y}=x\left(1+\left(R_{1}+R_{3}\right) x^{2} y+\left(R_{1}-3 R_{3}\right) y^{3}\right)$.

Polar coordinates:

$\dot{r}=r^{4}\left(R_{1} \cos \varphi+R_{3} \cos 3 \varphi\right)$,

$$
\dot{\varphi}=1 \text {. }
$$

Strong first integral:

$$
H=\frac{\left(x^{2}+y^{2}\right)^{3}}{(U(x, y))^{2}}
$$

$$
\text { where }
$$

$U(x, y)=1+3\left(R_{1}+R_{3}\right) x^{2} y+$ $+\left(3 R_{1}-R_{3}\right) y^{3}$.

Reciprocal integrating factor:

$$
V=\left(x^{2}+y^{2}\right) U(x, y) \text {. }
$$

Linearization:

$$
\chi=\frac{x}{(U(x, y))^{\frac{1}{3}}}, \eta=\frac{y}{(U(x, y))^{\frac{1}{3}}} .
$$

Transversal commuting system:

$$
\begin{aligned}
& \dot{x}=x U(x, y), \\
& \dot{y}=y U(x, y) .
\end{aligned}
$$

\section{System H42}

Cartesian coordinates:

$$
\begin{gathered}
\dot{x}=-y-\frac{4}{9} x^{4}-\frac{40}{9} x^{2} y^{2}+\frac{4}{3} y^{4}, \\
\dot{y}=x+\frac{20}{9} x^{3} y-\frac{28}{9} x y^{3} .
\end{gathered}
$$

Polar coordinates:

$\dot{r}=r^{4}\left(\frac{1}{3} \cos 3 \varphi-\frac{7}{9} \cos \varphi\right)$,

$\dot{\varphi}=1+r^{3}\left(\sin 3 \varphi-\frac{1}{3} \sin \varphi\right)$.

$$
\begin{gathered}
H(x, y)=\frac{\text { Strong first integral: }}{x^{2}+y^{2}} \\
\begin{array}{c}
U(x, y)^{\frac{1}{6}}+\frac{64\left(x^{2}+y^{2}\right)}{3} \int x y^{2}(U(x, y))^{-\frac{5}{6}} d x \\
\text { where }
\end{array} \\
-24 y^{3}+32 x^{2} y^{4}+16 y^{6}
\end{gathered}
$$

Reciprocal integrating factor:

$$
V(x, y)=\left(x^{2}+y^{2}\right)^{2}(U(x, y))^{\frac{5}{6}} \text {. }
$$

Transversal commuting system:

$$
\dot{x}=x\left(3-16 y^{3}\right)\left(x^{2}+y^{2}\right)(U(x, y))^{-\frac{1}{6}},
$$

$\dot{y}=y\left(3+12 x^{2} y-4 y^{3}\right)\left(x^{2}+y^{2}\right)(U(x, y))^{-\frac{1}{6}}$ 
TABLE 13.

Cubic reversible system (II).

\begin{tabular}{||c|c||}
\hline System H43 & System H4 4 \\
Cartesian coordinates: & Cartesian coordinates: \\
$\dot{x}=-y-\frac{16}{9} x^{4}-\frac{28}{9} x^{2} y^{2}+\frac{4}{3} y^{4}$, & $\dot{x}=-y+\frac{4}{9} x^{4}-\frac{20}{9} x^{2} y^{2}$, \\
$\dot{y}=x+\frac{8}{9} x^{3} y-\frac{16}{9} x y^{3}$. & $\dot{y}=x+\frac{40}{9} x^{3} y+\frac{16}{9} x y^{3}$. \\
Polar coordinates: & Polar coordinates: \\
$\dot{r}=r^{4}\left(-\frac{1}{3} \cos 3 \varphi-\frac{13}{9} \cos \varphi\right)$, & $\dot{r}=r^{4}\left(-\frac{1}{3} \cos 3 \varphi+\frac{7}{9} \cos \varphi\right)$, \\
$\dot{\varphi}=1+r^{3}\left(\sin 3 \varphi-\frac{1}{3} \sin \varphi\right)$. & $\dot{\varphi}=1+r^{3}(\sin 3 \varphi+\sin \varphi)$. \\
Strong first integral: & Strong first integral: \\
$H=\frac{\left(x^{2}+y^{2}\right)^{3} U_{2}}{U_{1}}$, where & $H(x, y)=\frac{\left(x^{2}+y^{2}\right)^{3} U_{2}}{U_{1}}$, where \\
$U_{1}(x, y)=-3+8 x^{2} y+8 y^{3}$, & $U_{1}(x, y)=9+24 y\left(x^{2}+y^{2}\right)+32 x^{2}\left(x^{2}+y^{2}\right)^{2}$, \\
$U_{2}(x, y)=9-24 y^{3}+16 x^{2} y^{4}+16 y^{6}$. & $U_{2}(x, y)=9+16 x^{6}+24 x^{2} y+16 x^{4} y^{2}$. \\
Reciprocal integrating factor: & \\
$V(x, y)=\left(x^{2}+y^{2}\right) U_{1} U_{2}$ & $\operatorname{Reciprocal~integrating~factor:}$ \\
Transversal commuting system: & $V(x, y)=\left(x^{2}+y^{2}\right) U_{1} U_{2}$ \\
$\dot{x}=x\left(3-8 x^{2} y-16 y^{3}\right) U_{1}(x, y)$, & \\
$\dot{y}=y\left(3+4 x^{2} y-4 y^{3}\right) U_{1}(x, y)$. & Transversal commuting system: \\
& $\dot{x}=x\left(3+8 x^{2} y\right) U_{1}(x, y)$, \\
\hline
\end{tabular}


TABLE 14.

Cubic reversible system (III).

\section{System H45}

Cartesian coordinates:

$$
\begin{gathered}
\dot{x}=-y+\left(r_{3}+R_{1}\right) x^{4}-\left(6 r_{3}+2 R_{1}\right) x^{2} y^{2}+\left(r_{3}-3 R_{1}\right) y^{4}, \\
\dot{y}=x+\left(4 R_{1}+4 r_{3}\right) x^{3} y+\left(4 R_{1}-4 r_{3}\right) x y^{3} .
\end{gathered}
$$

Polar coordinates:

$$
\dot{r}=r^{4}\left(r_{3} \cos 3 \varphi+R_{1} \cos \varphi\right)
$$$$
\dot{\varphi}=1+r^{3}\left(r_{3} \sin 3 \varphi+3 R_{1} \sin \varphi\right) \text {. }
$$

Strong first integral:

$H(x, y)=\frac{\left(x^{2}+y^{2}\right)^{3}}{U(x, y)}$ where $U(x, y)=1+\left(6 R_{1}+6 r_{3}\right) x^{2} y+\left(6 R_{1}-2 r_{3}\right) y^{3}$.

This first integral in polar coordinates is $H(r, \varphi)=\frac{r^{6}}{1+2 Q(\varphi) r^{3}}$.

From $H(r, \varphi)=C$, where $C$ is an arbitrary real constant different from zero, we can write $r^{3}=\frac{Q(\varphi) \pm \sqrt{Q(\varphi)^{2}+C}}{C}$.

From the differential equation $\dot{\varphi}=1+r^{3} Q(\varphi)$ we obtain

$$
T(r)=\int_{0}^{2 \pi} \frac{d \varphi}{1+r^{3} Q(\varphi)}=\int_{0}^{2 \pi}\left(1 \pm \frac{Q(\varphi)}{\sqrt{Q(\varphi)^{2}+C}}\right) d \varphi=2 \pi \pm \int_{0}^{2 \pi} \frac{Q(\varphi) d \varphi}{\sqrt{Q(\varphi)^{2}+C}}=2 \pi
$$

It is easy to see that the last integral is zero making the change $\omega=-\varphi$ and using the periodicity of $Q(\varphi)$. 
TABLE 15.

Cubic reversible system (IV).

\section{System $\mathrm{H}_{6}$}

Cartesian coordinates:

$\dot{x}=-y, \dot{y}=x+4 x^{3} y+4 x y^{3}$.

Polar coordinates:

$\dot{r}=r^{4}(-\cos 3 \varphi+\cos \varphi), \dot{\varphi}=1+r^{3}(\sin 3 \varphi+\sin \varphi)$.

For this system we could not find an analytic first integral but we have found an invariant of the form

$$
3 \varphi+4 r^{3} \cos ^{3} \varphi=3 t+C
$$

where $C$ is an arbitrary real constant.

From this invariant we can express the time in function of the variables $(r, \varphi)$ as follows

$$
t=\varphi+\frac{4}{3} r^{3} \cos ^{3} \varphi-\frac{C}{3} .
$$

If we recall that this system has a center

at the origin and therefore $r(0)=r(2 \pi)$

in a certain neighbourhood of the origin, we obtain

$t(2 \pi)-t(0)=\left[2 \pi+\frac{4}{3} r(2 \pi)-\frac{C}{3}\right]-\left[\frac{4}{3} r(0)-\frac{C}{3}\right]=2 \pi$.

TABLE 16.

Possible isocronous cubic reversible system.

\section{System $\mathbf{H}_{7}$}

Cartesian coordinates:

$\dot{x}=-y-\frac{\left(9-5 r_{3}\right)^{2}}{540} x^{4}+\frac{\left(3+r_{3}\right)\left(5 r_{3}-201\right)}{54} x^{2} y^{2}+\frac{5\left(3+r_{3}\right)^{2}}{36} y^{4}$, $\dot{y}=x+\frac{\left(81-5 r_{3}\right)\left(5 r_{3}-9\right)}{135} x^{3} y-\frac{5\left(3+r_{3}\right)^{2}}{27} x y^{3}$.

Polar coordinates:

$\dot{r}=r^{4}\left[\cos 5 \varphi+\frac{48+5 r_{3}}{45} \cos 3 \varphi+\frac{-1197+30 r_{3}-25 r_{3}}{540} \cos \varphi\right]$, $\dot{\varphi}=1+r^{3}\left[-\sin 5 \varphi+r_{3} \sin 3 \varphi+\frac{-9+6 r_{3}-5 r_{3}^{2}}{36} \sin \varphi\right]$. 
solutions of degree 3 and for $r_{3}=\frac{41}{5}, r_{3}=-\frac{45}{7}$ and $r_{3}=-\frac{87}{5}$ there are algebraic solutions of degree 6 . For the case $r_{3}=-\frac{87}{5}$ the system have a reciprocal integrating factor of the form $V(x, y)=M_{1}(x, y)^{\frac{2}{3}} M_{2}(x, y)^{\frac{5}{6}}$ where $M_{1}(x, y)$ and $M_{2}(x, y)$ are polynomials of degree 3 and 6 , respectively.

For system $\mathrm{H}_{2}$, the transversal commuting system has been found using particular solutions of the system.

All the systems listed in sections 10,11 and 12 are reversible. For some time it was an open question whether every polynomial system with an isochronous center has such a property (see Mardešić, Rousseau and Toni [68]). Chavarriga, Giné and García gave a counterexample in [19]. Such a system is not a uniformly isochronous system. The counterexample is a consequence of the following theorem which gives sufficient conditions for system (78) to have a center. The theorem is due to Chavarriga and Giné [15].

THEOREM 13.2. The origin of system (78) is a center in the following cases

(i) $\bar{\varphi}_{1}=\varphi_{1}, \bar{\varphi}_{3}=\varphi_{3}, 5 R_{3}+3 r_{3}=0$ and $5 R_{1}+r_{1}=0$.

(ii) $\bar{\varphi}_{1}=\varphi_{1}, \bar{\varphi}_{3}=\varphi_{3}, \varphi_{5}=5 \varphi_{1}$ and $\varphi_{3}=3 \varphi_{1}$.

(iii) $\bar{\varphi}_{1}=\varphi_{1}, \bar{\varphi}_{3}=\varphi_{3}, \varphi_{5}=2 \varphi_{1}+\varphi_{3}, r_{3}=3 R_{3}, r_{1}=2 R_{1}, R_{5}=R_{3}$ and $\left|R_{1}\right|=2\left|R_{3}\right|$.

(iv) $\bar{\varphi}_{1}=\varphi_{1}, \bar{\varphi}_{3}=\varphi_{3}, R_{5}=0$ and $\left\{\begin{array}{cc}(\text { iv.1 }) & R_{3} r_{1}-3 r_{3} R_{1}=0, \\ \text { (iv.2) } & r_{1}=3 R_{1} \text { and } r_{3}=-3 R_{3}, \\ \text { (iv.3) } & r_{1}=R_{1}=\left|3 R_{3}\right| \text { and } r_{3}=-3 R_{3} .\end{array}\right.$

In Theorem 13.1 case (ii) of theorem 13.2 has been studied.

As for the other cases considered in theorem 13.2, the only non-reversible isochronous center appears in case (iv.1), with $r_{1}=3 R_{1}$ (see system $H 4_{\alpha}$, next table).

In the other cases either there are no isochronous centers, or particular cases of previous theorems or trivial cases.

13.3. Isochronous centers of a linear center perturbed by homogeneous polynomials of degree 5 .

For $s=5$ system (75) takes the form

$$
\dot{r}=P_{5}(\varphi) r^{5}, \quad \dot{\varphi}=1+Q_{5}(\varphi) r^{4},
$$


TABLE 17.

Quartic homogeneous non-reversible isochronous center.

\section{System H4}

Cartesian coordinates:

$\dot{x}=-y+\left(k_{1} R_{1}+r_{3}\right) x^{4}-4 k_{2} R_{1} x^{3} y-\left(2 k_{1} R_{1}+6 r_{3}\right) x^{2} y^{2}-4 k_{2} R_{1} x y^{3}+\left(r_{3}-3 k_{1} R_{1}\right) y^{4}$ $\dot{y}=x+3 k_{2} R_{1} x^{4}+\left(4 k_{1} R_{1}+4 r_{3}\right) x^{3} y+2 k_{2} R_{1} x^{2} y^{2}+\left(4 k_{1} R_{1}-4 r_{3}\right) x y^{3}-k_{2} R_{1} y^{4}$.

where $k_{1}=\cos \varphi_{1}$ and $k_{2}=\sin \varphi_{1}$

Polar coordinates:

$\dot{r}=r^{4}\left(r_{3} \cos 3 \varphi+R_{1} \cos \left(\varphi+\varphi_{1}\right)\right)$,

$\dot{\varphi}=1+r^{3}\left(r_{3} \sin 3 \varphi+3 R_{1} \sin \left(\varphi+\varphi_{1}\right)\right)$.

Strong first integral:

$H=\frac{\left(x^{2}+y^{2}\right)^{3}}{1+6 k_{2} R_{1} x^{3}+6\left(k_{1} R_{1}+r_{3}\right) x^{2} y+6 k_{2} R_{1} x y^{2}+2\left(3 k_{1} R_{1}-r_{3}\right) y^{3}}$.

Reciprocal integrating factor:

$V=\left(x^{2}+y^{2}\right)\left(1+6 k_{2} R_{1} x^{3}+\left(6 k_{1} R_{1}+6 r_{3}\right) x^{2} y+6 k_{2} R_{1} x y^{2}+\left(6 k_{1} R_{1}-2 r_{3}\right) y^{3}\right)$.

The strong first integral in polar coordinates takes the form $H(r, \varphi)=\frac{r^{6}}{1+2 Q(\varphi) r^{3}}$, where $Q(\varphi)=3 R_{1} \sin \left(\varphi+\varphi_{1}\right)+r_{3} \sin 3 \varphi$.

From $H(r, \varphi)=1 / C$, where $C$ is an arbitrary real

constant different from zero, we can express $r^{3}$ as follows

$$
r^{3}=\frac{Q(\varphi) \pm \sqrt{Q(\varphi)^{2}+C}}{C} .
$$

From the differential equation $\dot{\varphi}=1+r^{3} Q(\varphi)$ we obtain

$$
T(r)=\int_{0}^{2 \pi} \frac{d \varphi}{1+r^{3} Q(\varphi)}=\int_{0}^{2 \pi}\left(1 \pm \frac{Q(\varphi)}{\sqrt{Q(\varphi)^{2}+C}}\right) d \varphi=2 \pi \pm \int_{0}^{2 \pi} \frac{Q(\varphi) d \varphi}{\sqrt{Q(\varphi)^{2}+C}}=2 \pi
$$

It is easy to see that the last integral is zero making the change $\omega=\varphi+\pi$ and using the periodicity of $Q(\varphi)$ 
where

$$
\begin{gathered}
P_{5}(\varphi)=R_{6} \cos \left(6 \varphi+\varphi_{6}\right)+R_{4} \cos \left(4 \varphi+\varphi_{4}\right)+R_{2} \cos \left(\varphi+\varphi_{2}\right)+R_{0} \\
Q_{5}(\varphi)=-R_{6} \sin \left(6 \varphi+\varphi_{6}\right)+r_{4} \sin \left(4 \varphi+\bar{\varphi}_{4}\right)+r_{2} \sin \left(\varphi+\bar{\varphi}_{2}\right)+r_{0}
\end{gathered}
$$

The reversibility conditions for system (80) are $R_{0}=0, \bar{\varphi}_{2}=\varphi_{2}, \bar{\varphi}_{4}=$ $\varphi_{4}, \varphi_{6}=3 \varphi_{2}$ and $\varphi_{4}=2 \varphi_{2}+\frac{\pi}{2}$. The following theorem, due to Chavarriga, Giné and García [20], characterizes reversible isochronous centers of system (80).

THEOREM 13.3. A necessary condition for the origin of system (78) to be a reversible isochronous center is that the system can be taken to one of the systems $\left(H 5_{1}\right),\left(H 5_{2}\right),\left(H 5_{3}\right),\left(H 5_{4}\right),\left(H 5_{5}\right),\left(\tilde{H} 5_{5}\right),\left(H 5_{6}\right),\left(H 5_{7}\right)$ and $\left(\tilde{H} 5_{7}\right)$ via a linear change of coordinates and a rescaling of time. The condition is sufficient for systems $\left(H 5_{1}\right),\left(H 5_{2}\right),\left(H 5_{3}\right),\left(H 5_{4}\right),\left(H 5_{5}\right)$, $\left(\tilde{H} 5_{5}\right)$ and $\left(H 5_{6}\right)$.

A transversal commuting system of system $H 5_{6}$ has been found using the same method used for system $\mathrm{H}_{2}$.

It is not known whether systems $H 5_{7}$ and $\tilde{H} 5_{7}$ have an isochronous center at $O$. Also no strong first integrals are known.

Analogously to what has been done in the case of quartic non-reversible isochronous centers, a class of nontrivial and non-reversible quintic isochronous centers can be obtained as a consequence of next integrability theorem, due to Chavarriga and Giné [16].

THEOREM 13.4. The origin of system (80) is a center in the following cases

(i) $R_{0}=0, \bar{\varphi}_{2}=\varphi_{2}, \bar{\varphi}_{4}=\varphi_{4}, 6 R_{4}+r_{4}=0$ and $6 R_{2}+2 r_{2}=0$.

(ii) $R_{0}=0, \bar{\varphi}_{2}=\varphi_{2}, \bar{\varphi}_{4}=\varphi_{4}, \varphi_{6}=3 \varphi_{2}$ and $\varphi_{4}=2 \varphi_{2}+\frac{\pi}{2}$.

(iii) $R_{0}=0, \bar{\varphi}_{2}=\varphi_{2}, \bar{\varphi}_{4}=\varphi_{4}, \varphi_{6}=\varphi_{4}+\varphi_{2}+\frac{\pi}{2}, r_{4}=R_{4}=R_{6}$, $r_{2}=R_{2}=r_{0}$, and $\left|R_{2}\right|=\left|R_{4}\right|$. 
TABLE 18.

Fifth degree homogeneous isochronous centers (I).

\section{System H5}

Cartesian coordinates:

$\dot{x}=-y+\left(4 R_{4}-2 R_{2}\right) x^{4} y-\left(2 R_{2}+4 R_{4}\right) x^{2} y^{3}$ $\dot{y}=x+\left(4 R_{4}-2 R_{2}\right) x^{3} y^{2}-\left(2 R_{2}+4 R_{4}\right) x y^{4}$.

Polar coordinates:

$\dot{r}=r^{5}\left(-R_{2} \sin 2 \varphi+R_{4} \sin 4 \varphi\right)$, $\dot{\varphi}=1$.

Strong first integral:

$$
H=\frac{\left(x^{2}+y^{2}\right)^{2}}{\left(U(x, y)^{2}\right)},
$$

where

$$
U(x, y)=1+4 R_{2} x^{4}+\left(4 R_{2}+8 R_{4}\right) x^{2} y^{2} .
$$

Reciprocal integrating factor:

$$
\begin{gathered}
V=\left(x^{2}+y^{2}\right) U(x, y) . \\
\text { Linearization: } \\
\chi=\frac{x}{(U(x, y))^{\frac{1}{4}}}, \eta=\frac{y}{(U(x, y))^{\frac{1}{4}}} .
\end{gathered}
$$

Transversal commuting system:

$$
\begin{aligned}
& \dot{x}=x U(x, y), \\
& \dot{y}=y U(x, y) .
\end{aligned}
$$

\section{System $\mathbf{H 5}_{2}$}

Cartesian coordinates:

$\dot{x}=-y+\frac{y}{2}\left(-9 x^{4}-13 x^{2} y^{2}+4 y^{4}\right)$,

$\dot{y}=x+\frac{x y^{2}}{2}\left(3 x^{2}-5 y^{2}\right)$.

Polar coordinates:

$\dot{r}=-r^{5}\left(-\frac{1}{2} \sin 4 \varphi-\frac{5}{4} \sin 2 \varphi\right)$,

$\dot{\varphi}=1+r^{4}(-\cos 4 \varphi+\cos 2 \varphi)$.

Strong first integral: $H=\frac{\left(x^{2}+y^{2}\right)^{2} U_{2}(x, y)}{\left(U_{1}(x, y)\right.}$, where

$U_{1}(x, y)=1-3\left(x^{2}+y^{2}\right) y^{2}$, $U_{2}(x, y)=1-4 y^{4}+4\left(x^{2}+y^{2}\right) y^{6}$.

Reciprocal integrating factor:

$$
V(x, y)=\left(x^{2}+y^{2}\right) U_{1} U_{2}
$$

Transversal commuting system: $\dot{x}=x\left(1-6 x^{2} y^{2}-10 y^{4}\right) U_{1}(x, y)$, $\dot{y}=y\left(1+2 x^{2} y^{2}-2 y^{4}\right) U_{1}(x, y)$. 
TABLE 19.

Fifth degree homogeneous isochronous centers (II).

\section{System $\mathbf{H 5}_{3}$}

Cartesian coordinates:

$\dot{x}=-y+y\left(-5 x^{4}+10 x^{2} y^{2}-y^{4}\right)$ $\dot{y}=x+x\left(x^{4}-10 x^{2} y^{2}+5 y^{4}\right)$.

Polar coordinates:

$\dot{r}=-r^{5} \sin 4 \varphi$,

$\dot{\varphi}=1+r^{4} \cos 4 \varphi$

Strong first integral:

$H=\frac{\left(x^{2}+y^{2}\right)^{4}}{1+2\left(x^{4}-6 x^{2} y^{2}+y^{4}\right)}$

Reciprocal integrating factor: $V=\left(x^{2}+y^{2}\right)\left(1+2\left(x^{4}-6 x^{2} y^{2}+y^{4}\right)\right)$.

Transversal commuting system: $\dot{x}=x+x\left(x^{4}-10 x^{2} y^{2}+5 y^{4}\right)$, $\dot{y}=y+y\left(5 x^{4}-10 x^{2} y^{2}+y^{4}\right)$.

\section{System $\mathbf{H 5}_{4}$}

Cartesian coordinates:

$\dot{x}=-y+x\left(x^{2}-5 y^{2}\right)\left(x^{2}+y^{2}\right)$, $\dot{y}=x+y\left(5 x^{2}-y^{2}\right)\left(x^{2}+y^{2}\right)$.

Polar coordinates: $\dot{r}=r^{5} \cos 2 \varphi$, $\dot{\varphi}=1+2 r^{4} \sin 2 \varphi$.

Strong first integral:

$$
H=\frac{\left(x^{2}+y^{2}\right)^{4}}{1+8 x y\left(x^{2}+y^{2}\right)} \text {. }
$$

Reciprocal integrating factor: $V=\left(x^{2}+y^{2}\right)\left(1+8 x y\left(x^{2}+y^{2}\right)\right)$.

Transversal commuting system: $\dot{x}=x+4 y\left(2 x^{2}-y^{2}\right)\left(x^{2}+y^{2}\right)$, $\dot{y}=y+4 x\left(2 y^{2}-x^{2}\right)\left(x^{2}+y^{2}\right)$. 
TABLE 20.

Fifth degree homogeneous isochronous centers (III).

\section{System $\mathbf{H 5}_{5}$}

Cartesian coordinates:

$\dot{x}=-y+\frac{y^{3}}{3}\left(-100 x^{2}+16 y^{2}\right)$,

$\dot{y}=x-\frac{20}{3} x y^{4}$.

Polar coordinates:

$\dot{r}=r^{5}\left(\sin 6 \varphi+\frac{1}{6} \sin 4 \varphi-\frac{10}{3} \sin 2 \varphi\right)$, $\dot{\varphi}=1+r^{4}\left(\cos 6 \varphi-\frac{8}{3} \cos 4 \varphi+\frac{5}{3} \cos 2 \varphi\right)$.

Strong first integral:

$$
\begin{gathered}
H=\frac{\left[x^{2}+y^{2}\left(1-\frac{16}{3} y^{4}\right)^{2}\right]^{2}}{(U(x, y))^{5}} . \\
\text { where } \\
U(x, y)=1-\frac{20}{3} y^{4} .
\end{gathered}
$$

Reciprocal integrating factor: $V=\left[x^{2}+y^{2}\left(1-\frac{16}{3} y^{4}\right)^{2}\right] U(x, y)$.

Linearization:

$$
\begin{gathered}
\chi=x(U(x, y))^{-\frac{5}{4}} \\
\eta=y\left[1-\frac{16}{3} y^{4}\right](U(x, y))^{-\frac{5}{4}}
\end{gathered}
$$

Transversal commuting system:

$$
\dot{x}=x\left(1-\frac{80}{3} y^{4}\right) U(x, y),
$$$$
\dot{y}=y\left(1-\frac{16}{3} y^{4}\right) U(x, y) \text {. }
$$

\section{System $\tilde{\mathbf{H}} 5_{5}$}

Cartesian coordinates:

$$
\begin{gathered}
\dot{x}=-y-\frac{20}{3} x^{4} y, \\
\dot{y}=x+\frac{x^{3}}{3}\left(16 x^{2}-100 y^{2}\right) .
\end{gathered}
$$

Polar coordinates:

$\dot{r}=r^{5}\left(\sin 6 \varphi-\frac{1}{6} \sin 4 \varphi-\frac{10}{3} \sin 2 \varphi\right)$, $\dot{\varphi}=1+r^{4}\left(\cos 6 \varphi+\frac{8}{3} \cos 4 \varphi+\frac{5}{3} \cos 2 \varphi\right)$.

Strong first integral:

$$
\begin{gathered}
H=\frac{\left[y^{2}+x^{2}\left(1+\frac{16}{3} x^{4}\right)^{2}\right]^{2}}{(U(x, y))^{5}} . \\
\text { where } \\
U(x, y)=1+\frac{20}{3} x^{4} .
\end{gathered}
$$

Reciprocal integrating factor: $V=\left[y^{2}+x^{2}\left(1+\frac{16}{3} x^{4}\right)^{2}\right] U(x, y)$.

$$
\begin{gathered}
\text { Linearization: } \\
\chi=x\left[1+\frac{16}{3} x^{4}\right](U(x, y))^{-\frac{5}{4}} \\
\eta=y(U(x, y))^{-\frac{5}{4}} .
\end{gathered}
$$

Transversal commuting system:

$$
\dot{x}=x\left(1+\frac{16}{3} x^{4}\right) U(x, y),
$$$$
\dot{y}=y\left(1+\frac{80}{3} x^{4}\right) U(x, y) \text {. }
$$ 
TABLE 21.

Fifth degree homogeneous isochronous centers (IV).

\section{System $\mathbf{H 5}_{6}$}

Cartesian coordinates:

$\dot{x}=-y+y\left(-x^{4}-4 x^{2} y^{2}+y^{4}\right), \dot{y}=x+2 x y^{2}\left(x^{2}-y^{2}\right)$.

Polar coordinates:

$\dot{r}=r^{5}\left(-\frac{1}{2} \sin 2 \varphi\right)$,

$\dot{\varphi}=1+r^{4}\left(-\frac{1}{2} \cos 4 \varphi+\frac{1}{2} \cos 2 \varphi\right)$.

Strong first integral:

$H=\frac{x^{2}+y^{2}}{U(x, y)}$, where $U(x, y)=4-\left(x^{2}+y^{2}\right) \ln \left[\frac{x^{2} y^{2}+\left(1+y^{2}\right)^{2}}{x^{2} y^{2}+\left(1-y^{2}\right)^{2}}\right]$

Reciprocal integrating factor:

$V=\left(x^{2}+y^{2}\right)^{2}\left(\left(1+y^{2}\right)^{2}+x^{2} y^{2}\right)\left(\left(1-y^{2}\right)^{2}+x^{2} y^{2}\right)$.

Transversal commuting system:

$\dot{x}=x\left(1-x^{2} y^{2}-5 y^{4}\right) U(x, y)$,

$\dot{y}=y\left(1+3 x^{2} y^{2}-y^{4}\right) U(x, y)$.

TABLE 22.

Possible fifth degree homogeneous isochronous centers.

\section{System H57}

Cartesian coordinates:

$\dot{x}=-y+\frac{4}{3} x^{2} y\left(4 x^{2}-3 y^{2}\right)$,

$\dot{y}=x+\frac{4}{3} x y^{2}\left(-8 x^{2}+9 y^{2}\right)$.

Polar coordinates:

$\dot{r}=r^{5}\left(\sin 6 \varphi-\frac{5}{6} \sin 4 \varphi \frac{4}{3} \sin 2 \varphi\right)$,

$\dot{\varphi}=1+r^{4}(\cos 6 \varphi-\cos 2 \varphi)$.

\section{System $\tilde{H}_{7}$}

Cartesian coordinates: $\dot{x}=-y+\frac{4}{3} x^{2} y\left(9 x^{2}-8 y^{2}\right)$, $\dot{y}=x+\frac{4}{3} x y^{2}\left(-3 x^{2}+4 y^{2}\right)$.

Polar coordinates: $\dot{r}=r^{5}\left(\sin 6 \varphi+\frac{5}{6} \sin 4 \varphi \frac{4}{3} \sin 2 \varphi\right)$, $\dot{\varphi}=1+r^{4}(\cos 6 \varphi-\cos 2 \varphi)$. 
(iv) $R_{0}=0, \bar{\varphi}_{2}=\varphi_{2}, \bar{\varphi}_{4}=\varphi_{4}, R_{6}=0$ and

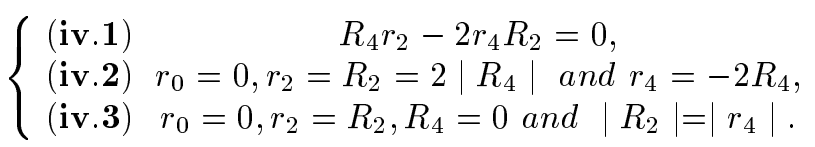

Analizing cases (iv.1) and (iv.3), two classes of non-trivial and nonreversible systems appear (see, respectively system $H 5_{\alpha}$ and $H 5_{\beta}$ ).

\section{ISOCHRONOUS CENTERS OF A CUBIC SYSTEM WITH DEGENERATE INFINITY}

We consider the system

$$
\dot{x}=-y+X_{2}(x, y)+X_{3}(x, y), \quad \dot{y}=x+Y_{2}(x, y)+Y_{3}(x, y),
$$

where $X_{s}(x, y)$ and $Y_{s}(x, y), s=2,3$ are homogeneous polynomials of degree $s$, satisfying $x Y_{3}(x, y)-y X_{3}(x, y) \equiv 0$ and $X_{3}^{2}+Y_{3}^{2} \not \equiv 0$. Such systems are a particular class of cubic systems with degenerate infinity. This name is due to the fact that, in Poincare compactification of (82), the line at infinity is filled with critical points (see Sotomayor [100]).

Similar systems have been studied by Chen Guang-Qing and Liang ZhaoJun [23] and recently by Gasull and Prohens [47]. In [47] an affine classification of quadratic systems with degenerate infinity is given. Yasmin [112] computes the Liapunov quantities for the origin of system (82) in order to find its maximum order, and the number of limit cycles that can be produced by a bifurcation. Christopher gives local first integrals or integrating factors for system (82) in cartesian coordinates. Chavarriga and Giné [17] show, for all integrable cases of these systems but one, which is a particular reversible cubic system, that there always exists a local analytical change of coordinates reducing these systems to integrable quadratic systems. This result implies, in particular, that all information one knows about the integrability of quadratic systems is applicable to the considered systems. This result justifies the name of a paper of Christopher, Devlin, Lloyd, Pearson and Yasmin [30] entitled Quadratic-like cubic systems.

In this section we list the isochronous centers of system (82). In the study of this problem polar coordinates have been used because this simplifies the computation of Liapunov constants. In Lemma 14.2 the expression of system (82) in polar coordinates is given. In Theorem 14.2 centers of 
TABLE 23.

Fifth degree homogeneous non-reversible isochronous centers (I).

\section{System $\mathbf{H 5} 5_{\alpha}$}

Cartesian coordinates:

$$
\begin{gathered}
\dot{x}=-y+\left(R_{2}+r_{4}\right) x^{5}-\left(4 R_{2}+10 r_{4}\right) x^{3} y^{2}+\left(5 r_{4}-5 R_{2}\right) x y^{4}, \\
\dot{y}=x+\left(5 R_{2}+5 r_{4}\right) x^{4} y+\left(4 R_{2}-10 r_{4}\right) x^{2} y^{3}+\left(r_{4}-R_{2}\right) y^{5} .
\end{gathered}
$$

Polar coordinates:

$\dot{r}=r^{5}\left(r_{4} \cos 4 \varphi+R_{2} \cos 2 \varphi\right)$,

$\dot{\varphi}=1+r^{4}\left(r_{4} \sin 4 \varphi+2 R_{2} \sin 2 \varphi\right)$.

$$
H=\frac{\text { Strong first integral: }}{\left(x^{2}+y^{2}\right)^{4}} \frac{}{1+8\left(R_{2}+r_{4}\right) x^{3} y+8\left(R_{2}-r_{4}\right) x y^{3}} .
$$

Reciprocal integrating factor:

$$
V=\left(x^{2}+y^{2}\right)\left(1+8\left(R_{2}+r_{4}\right) x^{3} y+8\left(R_{2}-r_{4}\right) x y^{3}\right) \text {. }
$$

This first integral in polar coordinates takes the form

$$
H(r, \varphi)=\frac{r^{8}}{1+2 Q(\varphi) r^{4}}, \text { where }
$$

$$
Q(\varphi)=2 R_{2} \sin 2 \varphi+r_{4} \sin 4 \varphi \text {. From } H(r, \varphi)=C,
$$

where $C$ is an arbitrary real constant different from zero,

$$
\text { we can express } r^{4} \text { like } r^{4}=\frac{Q(\varphi) \pm \sqrt{Q(\varphi)^{2}+C}}{C} \text {. }
$$

From the differential equation $\dot{\varphi}=1+r^{4} Q(\varphi)$ we obtain

$T(r)=\int_{0}^{2 \pi} \frac{d \varphi}{1+r^{4} Q(\varphi)}=\int_{0}^{2 \pi}\left(1 \pm \frac{Q(\varphi)}{\sqrt{Q(\varphi)+C}}\right) d \varphi=2 \pi \pm \int_{0}^{2 \pi} \frac{Q(\varphi) d \varphi}{\sqrt{Q(\varphi)^{2}+C}}=2 \pi$

It is easy to see that the last integral is zero making the change $\omega=-\varphi$ and using the periodicity of $Q(\varphi)$. 
TABLE 24.

Fifth degree homogeneous non-reversible isochronous centers (II).

System H5 $\mathbf{H}_{\beta}$
Cartesian coordinates:
$\dot{x}=-y-4 k_{1} k_{2} x^{5}+\left(4-8 k_{1}^{2}+4 k_{2}^{2}\right) x^{4} y+8 k_{1} k_{2} x^{3} y^{2}-$
$-\left(8+8 k_{1}^{2}\right) x^{2} y^{3}+12 k_{1} k_{2} x y^{4}+\left(4-4 k_{2}^{2}\right) y^{5}$,
$\dot{y}=x+\left(4 k_{1}^{2}-4\right) x^{5}-12 k_{1} k_{2} x^{4} y+\left(8+8 k_{2}^{2}\right) x^{3} y^{2}-$
$-8 k_{1} k_{2} x^{2} y^{3}-\left(4+4 k_{1}^{2}-8 k_{2}^{2}\right) x y^{4}+4 k_{1} k_{2} y^{5}$.
where $k_{1}=\cos \omega, k_{2}=\sin \omega$.
Polar coordinates:
$\dot{r}=-2 r^{5} \sin (2 \varphi+2 \omega)$,
Strong first integral:
$x^{2}+y^{2}$


system (82) are characterized. Lemma 14.2 and Theorem 14.2 are due to Chavarriga and Giné [17]. See also Sáez and Szántó [91].

LEMMA 14.2. In polar coordinates $x=r \cos (\varphi), y=r \sin (\varphi)$ system (82) has the form

$$
\dot{r}=P_{2}(\varphi) r^{2}+P_{3}(\varphi) r^{3}, \quad \dot{\varphi}=1+Q_{2}(\varphi) r
$$

where $P_{2}(\varphi), Q_{2}(\varphi)$ and $P_{3}(\varphi)$, are trigonometric polynomials of the form

$$
\begin{aligned}
& P_{2}(\varphi)=a_{3} \cos 3 \varphi+a_{1} \cos \varphi+a_{2} \sin \varphi \\
& Q_{2}(\varphi)=-a_{3} \sin 3 \varphi+b_{1} \cos \varphi+b_{2} \sin \varphi \\
& P_{3}(\varphi)=c_{0}+c_{1} \cos 2 \varphi+c_{2} \sin 2 \varphi
\end{aligned}
$$

THEOREM 14.1. For all integrable cases of system (83) there is a local analitycal change of coordinates in a neighbourhood of the origin such that, after an analytic reparametrization of time, the cubic system with degenerate infinity has one of the following expressions in polar coordinates:

$$
\begin{aligned}
& \text { (i) } \dot{r}=\left(R_{3} \sin 3 \varphi+R_{1} \sin \varphi\right) r^{2}+R_{2} \sin 2 \varphi r^{3}, \dot{\varphi}=1+R_{3} \cos 3 \varphi r ; \\
& \text { (ii) } \dot{r}=\left(R_{3} \cos 3 \varphi+R_{1} \cos \left(\varphi+\varphi_{1}\right)\right) r^{2}, \dot{\varphi}=1-\left(R_{3} \sin 3 \varphi+3 R_{1} \sin (\varphi+\right. \\
& \left.\left.\varphi_{1}\right)\right) r \\
& \text { (iii) } \dot{r}=R_{3}\left(\cos 3 \varphi \pm 3 \cos \left(\varphi+\varphi_{1}\right)\right) r^{2}, \dot{\varphi}=1+R_{3}\left(-\sin 3 \varphi \pm \sin \left(\varphi+\varphi_{1}\right)\right) r \\
& \text { (iv) } \dot{r}=\left(R_{3} \cos 3 \varphi+R_{1} \cos \left(\varphi+\varphi_{1}\right)\right) r^{2}, \dot{\varphi}=1+\left(-R_{3} \sin 3 \varphi+R_{1} \sin (\varphi+\right. \\
& \left.\left.\varphi_{1}\right)\right) r \text {. }
\end{aligned}
$$

The following theorem, which characterizes isochronous centers of cubic systems with degenerate infinity, is due Chavarriga, García and Giné [18].

THEOREM 14.2. A necessary and sufficient condition for the origin of system (82) to be an isochronous center is that the system can be brought to one of the following systems $\left(C D I_{1}\right),\left(C D I_{2}\right),\left(C D I_{3}\right),\left(C D I_{4}\right),\left(S_{2}^{*}\right)$, (see Theorem 26), by a linear change of coordinates and a rescaling of time. 
TABLE 25 .

Isocronous cubic systems with degenerate infinity (I).

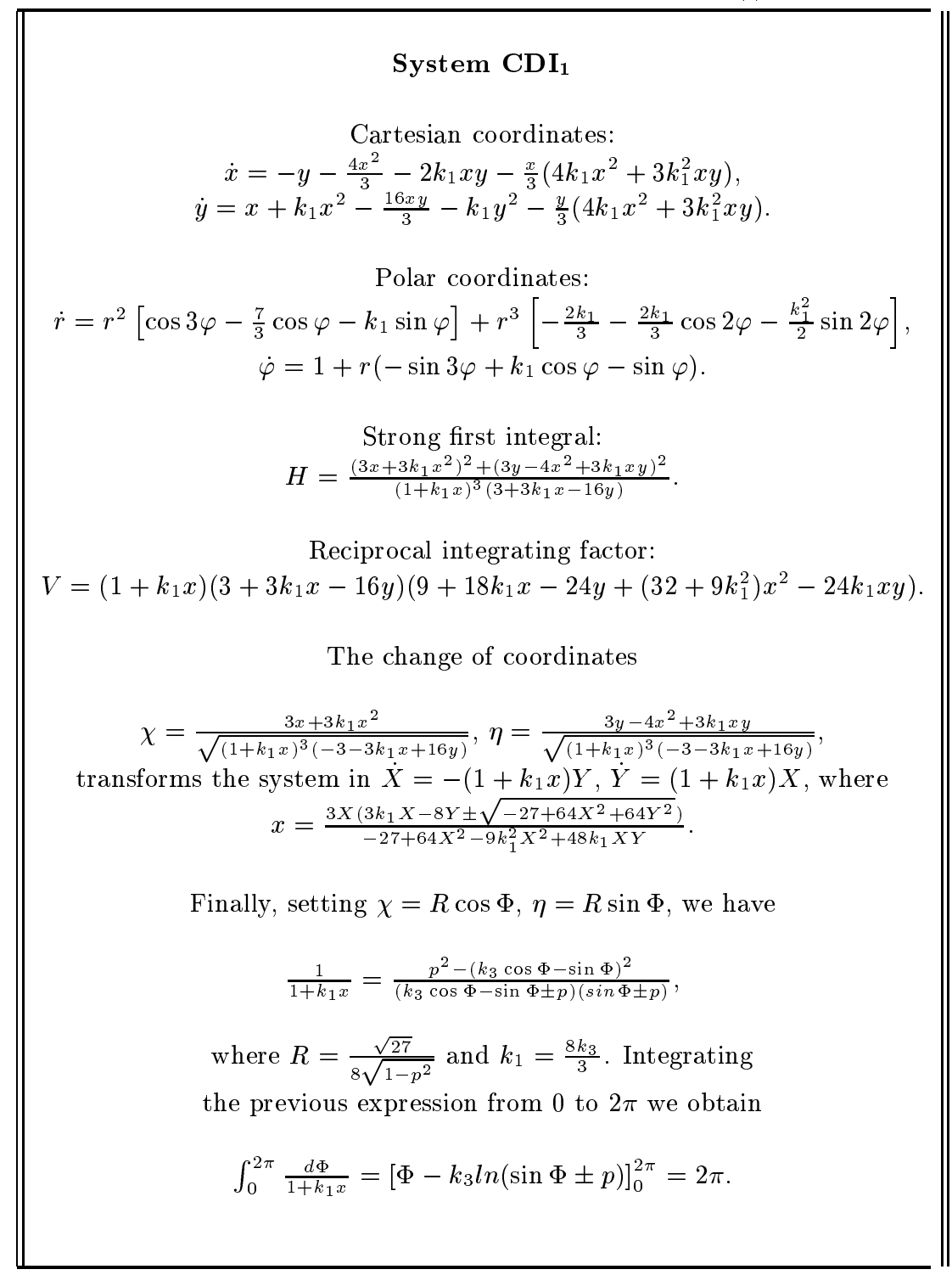


TABLE 26.

Isocronous cubic systems with degenerate infinity (II).

\section{System $\mathrm{CDI}_{2}$}

Cartesian coordinates:

$$
\begin{gathered}
\dot{x}=-y+\frac{16 x^{2}}{3}-2 k_{1} x y-\frac{4 y^{2}}{3}+\frac{k_{1} x}{3}\left(16 x^{2}-3 k_{1} x y-4 y^{2}\right), \\
\dot{y}=x+k_{1} x^{2}+\frac{8 x y}{3}-k_{1} y^{2}+\frac{k_{1} y}{3}\left(16 x^{2}-3 k_{1} x y-4 y^{2}\right) .
\end{gathered}
$$

Polar coordinates:

$$
\begin{gathered}
\dot{r}=r^{2}\left[\cos 3 \varphi+\frac{13}{3} \cos \varphi-k_{1} \sin \varphi\right]+r^{3}\left[2 k_{1}+\frac{10 k_{1}}{3} \cos 2 \varphi-\frac{k_{1}^{2}}{2} \sin 2 \varphi\right], \\
\dot{\varphi}=1+r\left(-\sin 3 \varphi+k_{1} \cos \varphi+\frac{1}{3} \sin \varphi\right) . \\
\text { Strong first integral: } \\
H=\frac{\left(3 x+3 k_{1} x^{2}\right)^{2}+\left(3 y+3 k_{1} x y+4 y^{2}\right)^{2}}{\left(3+3 k_{1} x+8 y\right)^{4}} .
\end{gathered}
$$

Reciprocal integrating factor:

$V=\left(1+k_{1} x\right)\left(3+3 k_{1} x+8 y\right)\left(9+18 k_{1} x+96 y+\left(9 k_{1}^{2}-256\right) x^{2}+96 k_{1} x y+128 y^{2}\right)$

The change of coordinates

$$
\chi=\frac{3 x+3 k_{1} x^{2}}{\left(3+3 k_{1} x+8 y\right)^{2}}, \eta=\frac{3 y+3 k_{1} x y+4 y^{2}}{\left(3+3 k_{1} x+8 y\right)^{2}}
$$

transforms the system in $\dot{X}=-\left(1+k_{1} x\right) Y, \dot{Y}=\left(1+k_{1} x\right) X$, where $x=\frac{3 X}{1-3 k_{1} X-16 Y}$.

Finally, setting $\chi=R \cos \Phi, \eta=R \sin \Phi$, we have

$$
\frac{1}{1+k_{1} x}=1+\frac{3 k_{1} R \cos \Phi}{16 R \sin \Phi-1} .
$$

Integrating the previous expression from 0 to $2 \pi$ we obtain

$$
\int_{0}^{2 \pi} \frac{d \Phi}{1+k_{1} x}=\left[\Phi+\frac{3 k_{1} \ln (16 R \sin \Phi-1)}{16}\right]_{0}^{2 \pi}=2 \pi .
$$


TABLE 27.

Isocronous cubic systems with degenerate infinity (III).

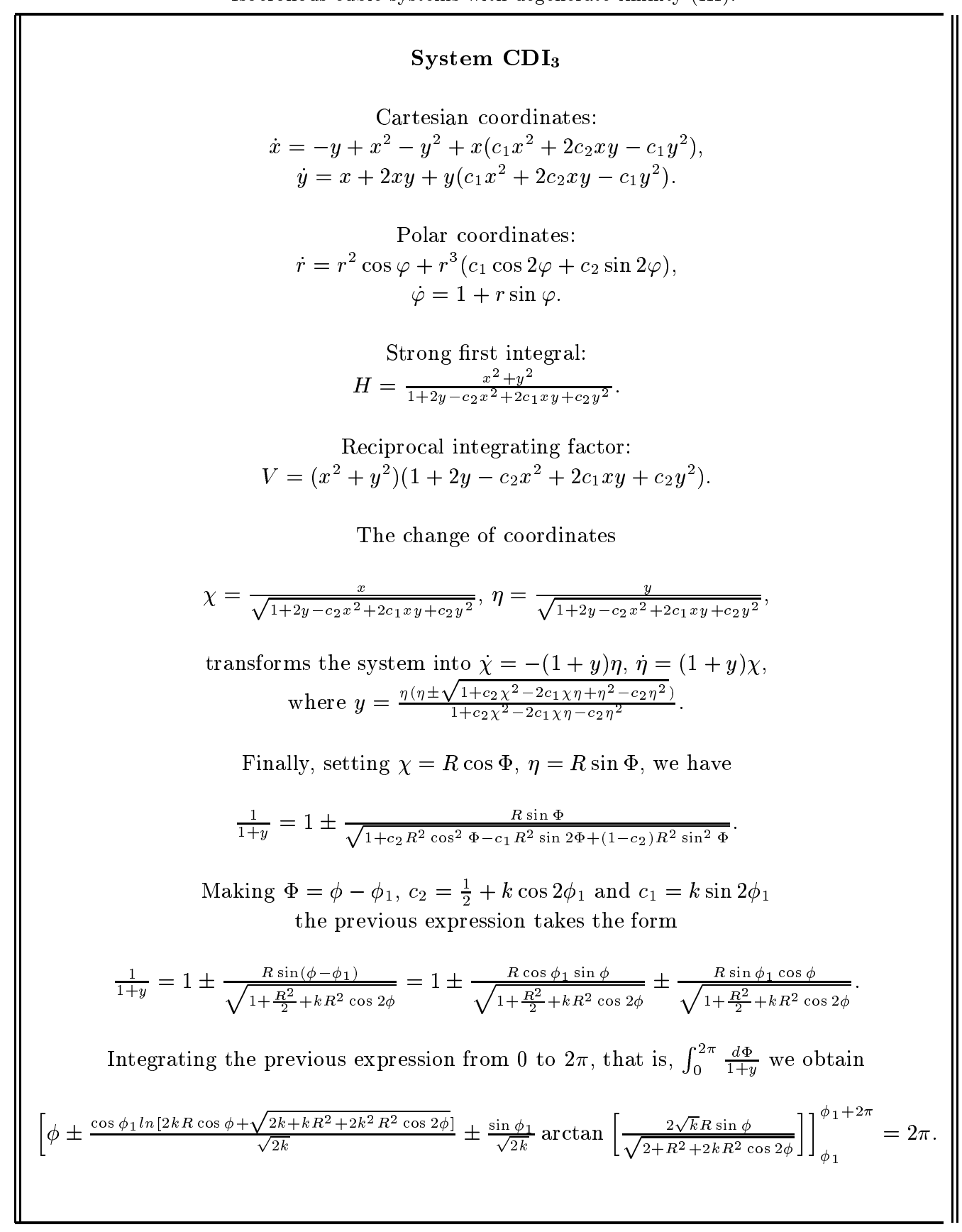


TABLE 28.

Isocronous cubic systems with degenerate infinity (IV).

\section{System $\mathrm{CDI}_{4}$}

Cartesian coordinates:

$$
\begin{gathered}
\dot{x}=-y+a_{1} x^{2}+a_{2} x y-a_{1} y^{2}+x\left(2 c_{2} x y+a_{1} a_{2} y^{2}\right), \\
\dot{y}=x+2 a_{1} x y+a_{2} y^{2}+y\left(2 c_{2} x y+a_{1} a_{2} y^{2}\right) .
\end{gathered}
$$

Polar coordinates:

$$
\begin{gathered}
\dot{r}=r^{2}\left(a_{1} \cos \varphi+a_{2} \sin \varphi\right)+r^{3}\left[\frac{a_{1} a_{2}}{2}-\frac{a_{1} a_{2}}{2} \cos 2 \varphi+c_{2} \sin 2 \varphi\right], \\
\dot{\varphi}=1+r a_{1} \sin \varphi .
\end{gathered}
$$

$$
\begin{gathered}
H=\frac{x^{2}+y^{2}}{\left(1-a_{2} x+2 a_{1} y+\left(a_{1}^{2}-2 c_{2}\right) x^{2}-a_{1} a_{2} x y+a_{1}^{2} y^{2}\right)}\left[\frac{2-\left(a_{2}-\sqrt{8 c_{2}-4 a_{1}^{2}+a_{2}^{2}}\right) x+2 a_{1} y}{2-\left(a_{2}+\sqrt{8 c_{2}-4 a_{1}^{2}+a_{2}^{2}}\right) x+2 a_{1} y}\right]^{\alpha}, \\
\text { if } 8 c_{2}-4 a_{1}^{2}+a_{2}^{2} \geq 0 \text { and } \alpha=\frac{a_{2}}{\sqrt{8 c_{2}-4 a_{1}^{2}+a_{2}^{2}}} . \\
H=\frac{x^{2}+y^{2}}{\left(1-a_{2} x+2 a_{1} y+\left(a_{1}^{2}-2 c_{2}\right) x^{2}-a_{1} a_{2} x y+a_{1}^{2} y^{2}\right)} \exp \left[\frac{2 a_{2} \arctan \left[\frac{2-a_{2} x+2 a_{1} y}{\sqrt{4 a_{1}^{2}-a_{2}^{2}-8 c_{2} x}}\right]}{\sqrt{4 a_{1}^{2}-a_{2}^{2}-8 c_{2}}}\right] .
\end{gathered}
$$

Reciprocal integrating factor:

$$
V=\left(x^{2}+y^{2}\right)\left(1-a_{2} x+2 a_{1} y+\left(a_{1}^{2}-2 c_{2}\right) x^{2}-a_{1} a_{2} x y+a_{1}^{2} y^{2}\right) \text {. }
$$

Transversal commuting system $\dot{x}=x-a_{2} x^{2}+2 a_{1} x y-x\left(2 c_{2} x^{2}+a_{1} a_{2} x y\right)$, $\dot{y}=y-a_{1} x^{2}-a_{2} x y+a_{1} y^{2}-y\left(2 c_{2} x^{2}+a_{1} a_{2} x y\right)$. 
TABLE 29.

Kukles isochronous systems.

System SK
Cartesian coordinates:
$\dot{x}=-y$,
$\dot{y}=x+3 x y+x^{3}$.
Polar coordinates:
$\dot{r}=\frac{3}{4}(\cos \varphi-\cos 3 \varphi) r^{2}+\frac{r^{3}}{8}(\sin 4 \varphi+2 \sin 2 \varphi)$,
$\dot{\varphi}=1+\frac{3 r}{4}(\sin \varphi+\sin 3 \varphi)+\frac{r^{2}}{8}(\cos 4 \varphi+4 \cos 2 \varphi+3)$.
Strong first integral:
$H=\frac{x^{2}+\left(x^{2}+y\right)^{2}}{\left(x^{2}+y+1\right)^{2}}$
Reciprocal integrating factor:
$V=\left(x^{2}+y+1\right)\left(\left(x^{2}+y\right)^{2}+x^{2}\right)$.
Linearization:
$\chi=\frac{x}{1+y+x^{2}}, \eta=\frac{x^{2}+y}{1+y+x^{2}}$.
Transversal commuting system:
$\dot{x}=x\left(1+y+x^{2}\right)$,
$\dot{y}=y-x^{2}+y^{2}-x^{4}$.

\section{KUKLES SYSTEM}

Let us consider Kukles' system

$$
\begin{aligned}
& \dot{x}=y, \\
& \dot{y}=-x+a_{1} x^{2}+a_{2} x y+a_{3} y^{2}+a_{4} x^{3}+a_{5} x^{2} y+a_{6} x y^{2}+a_{7} y^{3} .
\end{aligned}
$$

The following theorem is due to Christopher and Devlin [28]. See also Rousseau and Toni [83], for the case $a_{7}=0$. Volokitin and Ivanov [106] studied the reducibility of higher degree systems to Kukles' system.

THEOREM 15.1. The origin is an isochronous center of system (84) if and only if the system can be brought into system (SK) (see next table) through a change of coordinates and a rescaling of time. 


\section{REFERENCES}

1. A. Algaba, E. Freire And E. Gamero, Hypernormal form for the Hopf-zero bifurcation, Internat. J. Bifur. Chaos Appl. Sci. Engrg. 8 (1998), 1857-1887.

2. A. Algaba, E. Freire And E. Gamero, Isochronicity via normal form, Preprint, Universidad de Sevilla.

3. V.V. AMELKIN Isochronism of a center for two-dimensional analytic differential systems Differential equations 13 (1977), 667-674.

4. A.A. Andronov, A.A. Vitt and S.E. Khaikin, Theory of Oscillators, Dover Publications, New York, 1966.

5. V.I. ARno'ld, Équations Différentielles Ordinaires, Éditions Mir, Moscou, 1974.

6. V.I. Arno'Ld, Mathematical Methods of Classical Mechanics, Springer-Verlag, New York, 1989

7. D. Bambusi, G. Cicogna, G. Gaeta and G. Marmo Normal forms, symmetry and linearization of dynamical systems J. Phys. A: Math. Gen. 31 (1998), 5065-5082.

8. H. BASS, E.H. CONNEll And D. WRight, The Jacobian Conjecture: reduction of degree and formal expansion of the inverse, Bull. Amer. Math. Soc. 7 (1982), 287330.

9. N.N. BAUtin, On the number of limit cycles which appear with the variation of coefficients from an equilibrium position of focus or center type, Mat. Sb. 30 (1952), 181-196 (in russian); Amer. Math. Soc. Transl. 100 (1954), 397-413.

10. L. CESARI, Asymptotic behaviour and stability problems in ordinary differential equations, Springer-Verlag, Berlin, 1963.

11. J. CHAVARRIGA, Integrable systems in the plane with a center type linear part, App. Math. 22 (1994), 285-309.

12. J. Chavarriga AND I.A. GARCÍA, Isochronous centers of cubic reversible systems, Lecture Notes in Physics 518, Dynamical Systems, Plasmas and Gravitation, Springer-Verlag (1999), 255-268.

13. J. Chavarriga, H. Giacomini and J. Giné, The null divergence factor, Publ. Mat. 41 (1997), 41-56.

14. J. Chavarriga, H. Giacomini, J. Giné and J. Llibre, On the integrability of two-dimensional flows. To appear in Journal of Differential Equations.

15. J. CHAVARRIGA AND J. GINÉ, Integrability of a linear center perturbed by fourth degree homogeneous polynomial, Publ. Mat. 40 (1996), 21-39.

16. J. Chavarriga And J. Giné, Integrability of a linear center perturbed by fifth degree homogeneous polynomial, Publ. Mat. 41 (1997), 335-356.

17. J. ChAVARRIGA AND J. GINÉ, Integrability of cubic systems with degenerate infinity, Differential Equations and Dynamical Systems. 6, 4 (1998), 425-438.

18. J. Chavarriga, J. Giné And I.A. García, Isochronous centers of cubic systems with degenerate infinity, Differential Equations and Dynamical Systems, 7, 1 (1999).

19. J. ChAVARRIGA, J. GINÉ AND I.A. GARCíA, Isochronous centers of a linear center perturbed by fourth degree homogeneous polynomial, Bulletin des Sciences Mathématiques, 123 (1999), 77-96.

20. J. Chavarriga, J. Giné AND I.A. GARCíA, Isochronous centers of a linear center perturbed by fifth degree homogeneous polynomial, Preprint, Universitat de Lleida.

21. J. Chavarriga, J. Llibre and J. Sotomayor, Algebraic solutions for polynomial systems with emphasis in the quadratic case, Expo. Math. 15 (1997), 161-173. 
22. J. Chazy, Sur la thèorie des centres, C. R. Acad. Sci. Paris 221 (1947), 7-10.

23. Chen GuANG-QING AND Liang ZHAO-Jun, Affine classification for the quadratic vector fields without the critical points at infinity, J. of Math. Anal. and Appl. (1993), $62-72$.

24. L.A. CherkAs, Conditions for a Liénard equation to have a center, Differential Equations 12 (1977), 201-206.

25. C. CHICONE, The monotonicity of the period function for planar Hamiltonian vector fields, J. Differential Equations 69 (1987), 310-321.

26. C. Chicone And F. Dumortier, A quadratic system with a nonmonotonic period function, Proc. of AMS 102 (1988), 706-710.

27. C. Chicone AND M. JACOBS, Bifurcation of critical periods for plane vector fields, Trans. Amer. Math. Soc. 312 (1989), 433-486.

28. C. Christopher AND J. Devlin, Isochronous centres in planar polynomial systems, SIAM Jour. Math. Anal. 28 (1997), 162-177.

29. C. Christopher, J. Devlin And N.G. Lloyd, On the classification of Liénard systems with amplitude-independent periods, Preprint.

30. C. Christopher, J. Devlin, N.G. Lloyd, J.M. Pearson and N. Yasmin, Quadratic-like cubic systems, Differential Equations and Dynamical Systems, 5, 3-4, (1997), 329-345.

31. A. Cima, A. Gasull, V. Mañosa And F. Mañosas, Algebraic properties of the Liapunov and period constants, Rocky Mount. Jour. Math. 27 (1997), 471-501.

32. A. Cima, F. Mañosas And J. Villadelprat, Isochronicity for several classes of Hamiltonian systems, Preprint, Universitat Autònoma de Barcelona (1998).

33. C.B. Collins, The period function of some polynomial systems of arbitrary degree, Diff. and Int. Eq. 9 (1996), 251-266.

34. C.B. Collins, Conditions for a centre in a simple class of cubic systems, Diff. and Int. Eq. 10 (1997), 333-356.

35. R. Conti, On isochronous centers of cubic systems, Revue Roum. de Math. Pures et Appl. XXXIX (1994), 295-301.

36. R. CONTI, Uniformly isochronous centers of polynomial systems in $\mathbf{R}^{2}$, Lecture Notes in Pure and Appl. Math. 152 (1994), 21-31. M. Dekker, New York.

37. R. Conti, Centers of planar polynomial systems. A review, To appear.

38. W. COPPEL AND L. GAVRILOV, The period function of a hamiltonian quadratic system, Diff. and Int. Eq. 6 (1993), 1357-1365.

39. G. Darboux, Mémoire sur les équations differéntielles algébriques du premier ordre et du premier degré, Bull. Sci. Math. (2) 2 (1878), 60-96.

40. J. DeVlin, Coexisting isochronous and nonisochronous centres, Bull. Lond. Math. Soc. 28 (1996), 495-500.

41. G.F.D. DufF, Limit cycles and rotated vector fields, Ann. Math. 57 (1953), 15-31.

42. H. Dulac, Détermination et intégration d'une certaine classe d'équations différentielles ayant pour point singulier un centre, Bull. Sci. Math. (2) 32 (1908), $230-252$.

43. M. Farkas, Periodic Motions, Applied Mathematical Sciences, 104 Springer Verlag, Berlin, (1994). 
44. M. Frommer, Über das Auftreten von Wirbeln und Strudeln (geschlossener und spiraliger Integralkurven) in der Umgebung rationalre Unbestimmtkeitssellen, Math. Ann. 109 (1934), 395-424.

45. A. Gasull, A. Guillamón and V. Mañosa, Centre and isochronicity conditions for systems with homogeneous nonlinearities, Proceedings of the $2^{\text {nd }}$ Catalan Days of Applied Mathematics (1995) edited by M. Sofonea and J.N. Corvellec. Perpignan (1996).

46. A. Gasull, A. Guillamón, V. Mañosa and F. Mañosas, The period function for Hamiltonian systems with homogeneous nonlinearities, J. Differential Equations 139 (1997), 237-260.

47. A. Gasull And R. Prohens, Quadratic and cubic systems with degenerate infinity, J. of Math. Anal. Appl. 198 (1996), 25-34.

48. A. Gasull And J. Torregrosa, Center problem for several differential equations via Cherkas method, J. of Math. Anal. Appl. 228 (1998), 322-343.

49. L. GAVRILOV Isochronicity of plane polynomial Hamiltonian systems, Nonlinearity 10 (1997), 433-448.

50. J. Gregor, Dynamical systems with regular hand-side, Pokroky MFA III (1958), $153-160$.

51. O. HAJEK, Notes on meromorphic dynamical systems, I, Czechoslovak Math. J. 16 (1966), 14-27.

52. O. HАJEK, Notes on meromorphic dynamical systems, II, Czechoslovak Math. J. 16 (1966), 27-35.

53. HSU AND SzE-BI, A remark on the period of the periodic solution in the LotkaVolterra system, Jour. Math. Anal. Appl. 95 (1983), 428-436.

54. M.C. IRwin, Smooth Dynamical Systems, Academic Press, London, 1980.

55. X. Jim AND D. WANG, On Kukles' conditions for the existence of a centre, Bull. London Math. Soc. 22 (1990), 1-4.

56. W. KAPTEYN, On the centra of the integral curves which satisfy differential equations of the first order and the first degree, Konikl, Akademie von Wetenschappen te Amsterdam, Proceedings of the Section of Science, 13.2 (1911), 1241-1252; 14.2 (1911), 1185-1195.

57. O.A. KONONOVA, On the isochronicity of the center of a system of nonlinear oscillations, (Russian) Vestnik Beloruss. Gos. Univ. Ser. I Fiz. Mat. Mekh. (1990), 73-74, 81.

58. I.S. KukLES, Sur quelques cas de distinction entre un foyer et un centre, Dokl. Akad. Nauk. SSSR 42 (1944), 208-211.

59. J.J. Levin And S.S. Shatz, Nonlinear oscillations of fixed period, Jour. Math. Anal. Appl. 7 (1963), 284-288.

60. M.A. Liapunov, Problème général de la stabilité du mouvement, Ann. of Math. Stud. 17, Princeton University Press, 1947.

61. Li Chengzhi, Two problems of planar quadratic systems, Sci. Sinica Ser. A26 (1983), 471-481.

62. N.G. LlOYD, Small amplitude limit cycles of polynomial differential equations, Lect. Notes in Maths. 1032, Ordinary differential Equations and Operators (1983), 346356 .

63. N.G. LlOYd AND J.M. PEARSON, Computing centre conditions for certain cubic systems, Journal of Computational and Applied Mathematics 40 (1992), 323-336. 
64. W.S. LouD, Behavior of the period of solutions of certain plane autonomous systems near centers, Contributions to Differential Equations 3 (1964), 21-36.

65. N.A. LuKASHEVICH, Isochronicity of center for certain systems of differential equations, Diff. Uravn. 1 (1965), 220-226.

66. V.A. LUNKEVICH AND K.S. SiBIRSKII, Integrals of a general quadratic differential system in cases of a center, Diff. Equations 18 (1982), 563-568.

67. P. Mardes̆ić, L. Moser-Jauslin and C. Rousseau, Darboux linearization and isochronous centers with a rational first integral, J. Differential Equations 134 (1997), 216-268.

68. P. MARdeS̆IĆ, C. Rousseau AND B. TONi, Linearization of isochronous centers, J. Differential Equations 121 (1995), 67-108.

69. L. MAZZI AND M. SABATINI, A characterization of centres via first integrals, J. Differential Equations 76 (1988), 222-237.

70. L. MAZZI AND M. SABATINI, Commutators and linearizations of isochronous centers, Preprint, Università degli studi di Trento, UTM 482 (1996), presented at the Symposium on Planar Vector Fields, Lleida, November 24-27, 1996. To appear in Rend. Acc. Lincei.

71. G. MEISTERS, Jacobian problems in differential equations and algebraic geometry, Rocky Mount. J. Math. 12 (1982) 679-705.

72. V.V. Nemitskij and V.V. Stepanov, Qualitative Theory of Differential Equations, Princeton Univ. Press, Princeton, 1960.

73. С. Ові, Analytical theory of nonlinear oscillations, VII, The periods of the periodic solutions of the equation $\ddot{x}+g(x)=0$, J. Math. Anal. Appl. 55 (1976), 295-301.

74. P.J. Olver, Applications of Lie Groups to Differential Equations, Springer-Verlag, New York, 1986.

75. Z. OPIAL, Sur les périodes des solutions de l'équation différentielle $x^{\prime \prime}+g(x)=0$, Ann. Polon. Math. 10 (1961), 49-72.

76. I.A. PleshKAN, A new method of investigating the isochronicity of a system of two differential equations, Differential Equations 5 (1969), 796-802.

77. I.I. PleShKAN, On the isochronicity of systems of two differential equations whose right-hand sides contain common factors, Differential Equations 23 (1987), 657-667.

78. H. Poincaré, Mémoire sur les courbes définies par les équations différentielles, J. de Mathématiques (3) 7 (1881), 375-422; 8 (1882), 251-296; Oeuvres de Henri Poincaré, vol. I, Gauthier-Villars, Paris 1951, 3-84.

79. H. Poincaré, Mémoire sur les courbes définies par les équations différentielles, J. de Mathématiques Pures et Appliquées (4) 1 (1885), 167-244; Oeuvres de Henri Poincaré, vol. I, Gauthier-Villars, Paris 1951, 95-114.

80. N.V. Pyzhkova, AND L.P. Cherenkova Necessary conditions for isochronicity of the center of a differential system with a nonlinearity of the third power, (Russian. English summary) Dokl. Akad. Nauk BSSR 29 (1985), 980-981, 1052.

81. F. Rothe, The periods of the Lotka-Volterra system, J. Reine Angew. Math. (1985), $355129-138$.

82. C. Rousseau And B. Toni, Local bifurcations in vector fields with homogeneous nonlinearities of the third degree, Canad. Math. Bull. (1993), 36 473-484.

83. C. Rousseau And B. TONI, Local bifurcations of critical periods in the reduced Kukles sytems, Can. J. Math. 49 (1997) 338-358. 
84. M. SABATINI, Qualitative analysis of commuting flows on two-dimensional manifolds, Proc. of the Conference Equadiff 95, Lisboa, World Scientific Publ. Co., 494497.

85. M. SABATINi, Quadratic isochronous centers commute, Preprint, Università degli studi di Trento, UTM 461 (1995) - To appear in Applicationes Mathematicae.

86. M. SABATINI, Characterizing isochronous centres by Lie brackets, Diff. Equations and Dyn. Syst. 5 (1997), 91-99.

87. M. Sabatini, Dynamics of commuting systems on two-dimensional manifolds, Ann. Mat. Pura Appl. (IV) CLXXIII (1997), 213-232.

88. M. SABATINI, A connection between isochronous Hamiltonian centres and the Jacobian Conjecture, Nonlinear Analysis, 34 (1998), 829-838.

89. M. Sabatini, The time of commuting systems (preliminary version), Preprint, Università degli studi di Trento (1996), presented at the Symposium on Planar Vector Fields, Lleida, November 24-27, 1996.

90. M. SABAtini, On the period function of Liśystems, J. Differential Equations 152 (1999), 467-487.

91. E. SÁEz AND I. SzÁNTó, One-parameter family of cubic Kolmogorov system with an isochronous center, Collect. Math. 48 (1997), 297-301.

92. G. SANSONE AND R. CONTI, Non-linear differential equations, Revised edition. International Series of Monographs in Pure and Applied Mathematics, Vol. 67, The Macmillan Co., New York 1964.

93. R. SCHAAF, A class of Hamiltonian systems with increasing periods, Jour. Reine Ang. Math. 363 (1985), 96-109.

94. B. Schuman, Sur la forme normale de Birkhoff et les centres isochrones, C. R. Acad. Sci. Paris 322 (1996), 21-24.

95. Shi Songling, A method of constructing cycles without contact around a weak focus, J. Differential Equations 41 (1981), 301-312.

96. K.S. SiBIRSKII, Algebraic invariants for differential equations and matrices, Kshiner Shtiint-sa, 1976 (in Russian).

97. K.S. SiBIRSKII, On the number of limit cycles in the neighbourhood of a singular point, Differential Equations 1 (1965), 36-47.

98. J. SoKULSKI, The beginning of classification of Darboux integrals for cubic systems with center.

99. J. Sotomayor, Liçóes de Equaçóes Diferenciais Ordinárias, IMPA, Rio de Janeiro, 1979.

100. J. SотомAYOR, Curvas definidas por equaçóes diferenciais no plano, IMPA, Rio de Janeiro, 1981.

101. M. URABE, Potential forces which yield periodic motions of a fixed period, Jour. Math. Mech. 10 (1961), 569-578.

102. M. URABE, The potential force yielding a periodic motion whose period is an arbitrary continuous function of the amplitude of the velocity, Arch. Rational Mech. and Anal. 11 (1962), 27-33.

103. J. Villadelprat, Index of vector fields on manifolds and isochronicity for planar hamiltonian differential systems, Ph. d. Thesis, U.A.B., Facultat de Ciències, Departament de Matemàtiques, Barcelona, 1999.

104. M. VILLARINI, Regularity properties of the period function near a centre of planar vector fields, Nonlinear Analysis 19 (1992), 787-803. 
105. M. Villarini, Smooth linearizations of centres, Preprint, Università degli studi di Firenze (1996).

106. E.P. VOLOKITIN AND V.V. IVANOV Isochronicity and commutation of polynomial vector fields Siberian Math. Journal 40 (1999), 23-38.

107. A.P. Vorob'Ev, On isochronous systems of two differential equations, Dokladi Akademii Nauk Belorusskoi SSR 7 (1963), 155-156 (in russian).

108. A.P. VOROB'EV, The construction of isochronous systems of two differential equations, Dokladi Akademii Nauk Belorusskoi SSR 7 (1963), 513-515 (in russian).

109. A.P. VOROB'EV, Qualitative investigation in the large of integral curves of isochronous systems of two differential equations, Diff. Uravn. 1 (1965), 439-441 (in russian).

110. A.P. VOROB'EV, Sufficiency conditions for isochronism of canonical systems of two differential equations, Diff. Uravn. 1 (1965), 582-584 (in russian).

111. J. WALDVOGEL, The period in the Volterra-Lotka predator-prey model, SIAM Jour. Numer. Anal. 20 (1983), 1264-1272.

112. N. YASMIN, Closed orbits of certain two-dimensional cubic systems, Ph. D. thesis University College of Wales, Aberystwyth, (1989).

113. G. ZAMPIERI, On the periodic oscillations of $x^{\prime \prime}=g(x)$, Journal of Differential Equations 78 (1989) 74-88.

114. H. ŻOLA̧DEK, Quadratic systems with center and their perturbations, J. Differential Equations 109 (1994), 223-273.

115. H. ŻOLA̧DEK, On certain generalization of the Bautin's Thorem, Nonlinearity 7 (1994), 273-279.

116. H. ŻOLA̧DEK, The classification of reversible cubic systems with center, Topological Methods in Nonlinear Analysis 4 (1994) 79-136. 